\title{
Electroweak stability and discovery luminosities for new physics
}

\author{
Kerem Cankoçak ${ }^{1}$, Durmuş Demir ${ }^{2}$, Canan Karahan ${ }^{1, a}$, Sercan Şen $^{3}$ \\ ${ }^{1}$ Physics Engineering Department, İstanbul Technical University, 34469 Maslak, Istanbul, Turkey \\ ${ }^{2}$ Faculty of Engineering and Natural Sciences, Sabancı University, 34956 Tuzla, Istanbul, Turkey \\ ${ }^{3}$ Physics Engineering Department, Hacettepe University, 06800 Beytepe, Ankara, Turkey
}

Received: 6 March 2020 / Accepted: 13 December 2020 / Published online: 23 December 2020

(C) The Author(s) 2020

\begin{abstract}
What is the luminosity needed for discovering new physics if the electroweak scale is to remain stable? In this work we study this question, with the pertinent example of a real singlet scalar which couples to the Higgs field at the renormalizable level. Observing that the electroweak scale remains stable if the two scalars couple in a see-sawic fashion through a mass-degeneracy-driven unification linkup among quartic couplings at a given scale, we show by detailed simulation studies of the $p p \rightarrow$ (singlet scalar) $\rightarrow Z Z \rightarrow 4 \ell$ channel that the HL-LHC, which is expected to deliver an integrated luminosity of $3 \mathrm{ab}^{-1}$, has no significant excess of signal over the background in the $800-2000 \mathrm{GeV}$ mass range. The FCC-hh, on the other hand, can discover scalars up to a mass of $870 \mathrm{GeV}$ with an integrated luminosity $20 \mathrm{ab}^{-1}$. Observation at $3 \sigma$ (discovery at $5 \sigma$ ) of a new scalar with a minimum mass $800 \mathrm{GeV}$ requires at least $2 \mathrm{ab}^{-1}\left(5.2 \mathrm{ab}^{-1}\right)$ integrated luminosity, showing that the new physics that does not destabilize the electroweak scale is accessible only at very high luminosities, and can be tested already in the early stages of the FCC-hh operation period.
\end{abstract}

\section{Introduction}

The standard model of elementary particles (SM), experimentally completed by the discovery of the Higgs boson at the ATLAS and CMS [1], has shown excellent agreement with all the available data so far [2]. The TeV domain seems to be devoid of any new particles beyond the SM spectrum [3]. The SM seems thus to continue to hold good up to energies well above a TeV.

This experimental fact poses a big challenge. The reason is that there are all sort of astrophysical (dark matter, dark photon), cosmological (dark energy, inflation) and other (neutrino masses, flavor, unification, etc.) indications that the $\mathrm{SM}$ has to be extended by new fields beyond the $\mathrm{TeV}[4,5]$.

a e-mail: ckarahan@itu.edu.tr (corresponding author)
These fields set the so-called physics-beyond-the-SM (BSM) sector.

The problem with the BSM phenomena is that they generally drag the SM towards their scales. Indeed, if the SM+BSM is valid up to a scale $\Lambda$ and if the Higgs boson couples with strength $\lambda_{h-B S M}$ to BSM fields of masses $M_{B S M}$ then its mass $m_{h}$ receives the correction

$\delta m_{h}^{2}=c_{h} \Lambda^{2}+\tilde{c}_{h} \lambda_{h-B S M} M_{B S M}^{2} \log \frac{M_{B S M}^{2}}{\Lambda^{2}}$

where $c_{h}$ and $\tilde{c}_{h}$ are $\mathcal{O}\left(10^{-2}\right)$ loop factors, and the HiggsBSM coupling $\lambda_{h-B S M}$ changes from portal to portal in view of the SM-BSM coupling channel:

$\lambda_{h-B S M} \propto \begin{cases}\lambda_{H S} & \text { for Higgs portal }: \lambda_{H S} H^{\dagger} H S^{\dagger} S \\ \lambda_{Z^{\prime} B}^{2} & \text { for hypercharge portal }: \lambda_{Z^{\prime} B}^{2} Z_{\mu \nu}^{\prime} B^{\mu \nu} \\ \lambda_{L N}^{2} & \text { for neutrino portal }: \lambda_{L N} \bar{L} H N+\text { h.c. }\end{cases}$

The lesson from the LHC experiments is that the UV completion that eliminates the $\Lambda^{2}$ term in (1) must do it with a sufficiently small $\lambda_{h-B S M}$ in order to keep $\delta m_{h}^{2} / m_{h}^{2}$ sufficiently small. It is in this sense that the known completions of the SM (supersymmetry, extra dimensions, compositeness, and their hybrids) are sidelined because their BSM sectors (superpartners in supersymmetry, Kaluza-Klein levels in extra dimensions, and technifermions in compositeness) necessitate $\lambda_{h-B S M} \simeq \lambda_{S M}$, where $\lambda_{S M}$ is a typical SM coupling. It turns out that heavier the BSM larger the shift in the Higgs boson mass, and stronger the exclusion limits on the known SM completions. In view of this LHC lesson, we do in the present work three things:

1. In Sect. 2 below we discuss in detail how an $L H C$-favored SM completion must be, and give symmergence [6,7] as a likely realization in which $c_{h} \Lambda^{2}$ part is eliminated (actually transmuted to curvature) by keeping $\tilde{c}_{h}$ sufficiently 
small. Therein we analyze how $\tilde{c}_{h}$ can be structured, and construct a linkup scheme in which the seesawic coupling in [7] is reproduced.

2. In Sects. 3 and 4, without loss of generality, focus and goal, we specialize to a BSM sector in which only a real singlet scalar interacts with the SM Higgs boson, and reveal there the implications of the see-sawic couplings of Sect. 2 in view of the electroweak stability. (In general, one should analyze all three portals in (2) but for showing the stability of the electroweak scale against the logarithmic corrections in (1) it proves beneficial to focus on a singlet scalar $S$ as the worst case because $S$ itself is a power-law UV sensitive field.)

3. Section 5 we study the collider phenomenology of the real SM-singlet scalar in Sect. 3, with emphasis on electroweak stability. There we attempt to answer this key question:

What energy and luminosity does it take to discover a singlet scalar if the electroweak scale is to remain stable?

To answer this question we perform a detailed analysis of the decay and production channels associated with the singlet scalar, and determine explicitly discovery luminosities at the HL-LHC and FCC-hh colliders via the low-background 4-lepton signal. Our analysis in Sect. 5 shows how LHC-favored SM completions like symmergence can reveal themselves at high luminosities.

In Sect. 6 we conclude.

\section{LHC-favored SM completion}

If the LHC results $[1,3]$ have taught us anything it is that the SM can be extended with a BSM sector (facilitating neutrino masses, baryogenesis, dark matter, inflation, etc.) naturally if SM-BSM couplings (like $\lambda_{h-B S M}$ in $\delta m_{h}^{2}$ ) are allowed to be small $\left(\lambda_{h-B S M} \ll \lambda_{S M}\right)$ and if the power-law UV sensitivities (like $c_{h} \Lambda^{2}$ in $\delta m_{h}^{2}$ ) are properly nullified. There are thus two sides of the problem, and both sides need to be properly addressed.

The nullification of the power-law UV sensitivities is achieved ordinarily in all the known UV completions. They are eradicated in supersymmetry, downsized to $\mathrm{TeV}$ in extra dimensions, and turned into curvature in symmergence (emergence of gravity in a way restoring gauge symmetries broken explicitly by the UV cutoff $\Lambda$ ). These UV sensitivities (like $c_{h} \Lambda^{2}$ in $\delta m_{h}^{2}$ in Eq. (1) above) are thus far from destabilizing the electroweak scale.
The logarithmic UV sensitivities (like the $\lambda_{h-B S M}$ term in $\delta m_{h}^{2}$ in Eq. (1) above) are highly nontrivial in that the known SM completions have $\lambda_{S M-B S M} \simeq \lambda_{S M}$, and never get into the hierarchic regime $\lambda_{S M-B S M} \ll \lambda_{S M}$. This is a serious problem because heavier the BSM larger the logarithmic part of $\delta m_{h}^{2}$ since it is proportional to $\lambda_{h-B S M} M_{B S M}^{2}$ and since all known UV completions necessitate $\lambda_{S M-B S M} \simeq \lambda_{S M}$. The logarithmic part poses thus as a serious hierarchy problem (causing the so-called little hierarchy problem [8-10]). It is in this sense that the LHC results started sidelining known UV completions like supersymmetry, extra dimensions and compositeness. They point to new SM completions which can work with $\lambda_{S M-B S M} \ll \lambda_{S M}$ instead of $\lambda_{S M-B S M} \simeq$ $\lambda_{S M}$ (characteristic to supersymmetry, extra dimensions and compositeness).

\subsection{Symmergence as the LHC-favored UV completion}

The focus in the present work is on the logarithmic quantum corrections, that is, on the little hierarchy problem posed, for instance, by the logarithmic part of $\delta m_{h}^{2}$ in (1). In other words, one gives weight to logarithmic corrections with the assumption that there exists some UV completion that does away with the power-law UV sensitivities [11-13]. It is, nevertheless, necessary to have a concrete UV completion in mind even if it may not phenomenologically be directly relevant for the analysis of the effects of the logarithmic corrections. There is currently one such UV completion: Symmergent gravity $[6,7,14]$. To have an idea what symmergence is and is not one notes first that, in the presence of the UV momentum cutoff $\Lambda$, the photon and the gluon acquire masses (with zero logarithmic parts)

$\delta m_{\gamma, g}^{2}=c_{\gamma, g} \Lambda^{2}$

which completely destruct the SM by breaking the color and the electric charge (CCB) [14-17]. (In a broader sense, these are gauge anomalies.) This quantum gauge invariance breaking can be prevented if the quadratic corrections $c_{g} \Lambda^{2}$ and $c_{\gamma} \Lambda^{2}$ are somehow neutralized.

The gauge boson mass corrections (4) are physical. They cannot be altered by any means simply because the cutoff $\Lambda$ is physical. It is physical because it refers to physical phenomena beyond the SM (like gravity and possible BSM physics). If gravity were absent, if there were no BSM physics, if it were just the SM alone then all loop integrals would turn into cutoff regularization integrals [18] with arbitrary cutoff $\Lambda$, and one would then be able to eradicate all power-law corrections (like (4) and (1)) simply by switching to dimensional regularization [19]. The essence of the problem is that gravity and BSM physics (needed for dark matter, strong $\mathrm{CP}$, baryogenesis, and various other phenomena) introduce new physical scales like $\Lambda$, and neutralization of the gauge symmetry-breaking corrections like (4) necessitates a new 
mechanism that complies with the SM as well as the BSM and gravity.

In regard to neutralization of the loop-induced gauge boson masses in (4), symmergence is a mechanism resulting from the observation that it should be possible to set up a covariance relation (just like the usual general covariance between the flat metric and the curved metric) between $\Lambda^{2}$ (which explicitly breaks Poincaré invariance of the flat spacetime on which QFTs like the SM are based) and spacetime curvature (which explicitly breaks Poincaré invariance as a built-in feature of the curved spacetime). This extended covariance, which rests on the Poincaré affinity between $\Lambda^{2}$ and curvature, is guided by gauge invariance (as discussed in detail in [7]). In other words, the proposed covariance between $\Lambda^{2}$ and curvature must be able to restore gauge invariance by transmuting the loop-induced gauge boson masses in (4) appropriately. This transmutation mechanism leads to symmergence $[7,14]$, with the following salient features:

1. It predicts existence of a BSM sector (of neutrinos, dark matter, ..., and maybe more),

2. It kills the gauge boson mass term (4) (restores gauge invariance) by transmuting $\Lambda^{2}$ into affine curvature,

3. It converts $c_{h} \Lambda^{2}$ part of (1) into Higgs-curvature coupling,

4. It converts $c_{S} \Lambda^{2}$ part of the corrections $\delta m_{S}^{2}$ to the BSM scalar masses into $S$-curvature couplings,

5. It leads to Einstein gravity, and finally,

6. It results in dimensionally-regularized SM+BSM in the curved spacetime.

What is left untouched by symmergence is the logarithmic part of (1). It is left untouched because $\log \Lambda^{2}$ does not break Poincaré invariance (it always leads to multiplicative corrections). It remains as a physical contribution to the Higgs boson mass. This remnant contribution might give the impression that symmergence makes no real progress concerning the electroweak stability. No! Actually it makes a pivotal progress. It does because it leaves couplings between the SM and the BSM unconstrained, that is, free to take any perturbative value, even the zero! (This feature rests on the fact that the gravitational scale is set by the supertrace of the SM+BSM mass-squareds, with no necessity of any couplings between the SM and the BSM. In other words, the SM and BSM do not have to interact.) This means that the SM-BSM couplings can be small enough (compared to the SM couplings) to suppress logarithmic corrections. This SM-BSM coupling scheme is something specific to symmergence. Indeed, contrastively,

$\lambda_{S M B S M} \simeq \lambda_{S M} \Longrightarrow$ only light BSMs with $m_{B S M} \gtrsim m_{h}$ are allowed in supersymmetry, extra dimensions and compositeness $[4,5]$ whereas

$\lambda_{S M B S M} \ll \lambda_{S M} \Longrightarrow$ heavy BSMs with $m_{B S M} \gg m_{h}$

are allowed in symmergence $[7,14]$. It thus turns out that the LHC can exclude sparticles (the BSM of supersymmetry), Kaluza-Klein levels (the BSM of extra dimensions) and technifermions (the BSM of compositeness) but not the BSM of the symmergence! In fact, the Higgs mass correction in (1) remains within the LHC bounds if the SM-BSM couplings obey the bound

$\lambda_{h-B S M} \lesssim \frac{m_{H}^{2}}{m_{B S M}^{2}}$

which is a seesawic relation between the mass parameters of the Higgs field $H\left(m_{h}^{2}=-2 m_{H}^{2}\right.$ in the SM) and the BSM fields. This seesawic coupling scheme, ensuring that heavier the BSM smaller its couplings to the SM, gives way to a novel approach to collider and other searches for the BSM physics.

It should be noted that stabilization of the SM against $\Lambda^{2}$ corrections has been approached via various mechanisms. They include classical conformal invariance [20-22], twin Higgs [23,24], cosmological relaxation [25, 26], Higgs frame [27], gravitational relaxation [28], large copies of the SM spectrum [29,30], and subtraction of $\Lambda^{2}$ terms [31]. The subtraction method [31] is particularly relevant in that it subtracts (absorbs into critical surface) $\Lambda^{2}$ terms, retains only $\log \Lambda$ terms (dimensional regularization), predicts no BSM sector, and leaves out gravity entirely. Symmergence [7], on the other hand, identifies $\Lambda^{2}$ terms with curvature by their Poincaré affinity, retains only $\log \Lambda$ terms (dimensional regularization), predicts existence of a BSM sector, and makes gravity emerge upon the SM+BSM. This clear distinction between the two approaches shows that symmergence could indeed be a factual UV completion of the SM.

\subsection{Mass-degeneracy-driven unification linkup}

The seesawic scheme in (7), an empirical relation based on the freedom in (6) enabled by symmergence, is a just-so relation. The thing is that it works. The problem is that there is no obvious symmetry principle that supports it. (This can be seen from the symmetry structures of the two-Higgs doublet models [32-35].) To this end, it proves convenient to specialize to a real BSM scalar $S$ of mass $m_{S}$. (The remaining BSM fields do not have to couple to the SM; they can stay with zero couplings to the SM fields. But if any of them couples to the SM Higgs its effects must be taken into account in view of the portals in (2).) The scalar $S$ couples to the SM Higgs 
field via the potential

$V_{H S}=m_{H}^{2} H^{\dagger} H+\lambda_{H}\left(H^{\dagger} H\right)^{2}+\frac{m_{S}^{2}}{2} S^{2}+\frac{\lambda S}{4} S^{4}+\frac{\lambda H S}{2} H^{\dagger} H S^{2}$

whose boundedness from below necessitates

$\lambda_{H}>0, \quad \lambda_{S}>0,16 \lambda_{H} \lambda_{S}-\lambda_{H S}^{2}>0$

as primary constraints on dimensionless couplings.

It follows from the potential (8) that the $\log \Lambda$ sensitivities (translated into dimensional regularization via the formal equivalence $\log \Lambda^{2}=1 / \epsilon-\gamma_{E}+1+\log 4 \pi Q^{2}$ ) lead to nontrivial $\overline{M S}$ corrections to the Higgs condensation parameter $m_{H}^{2}\left(m_{h}^{2}=-2 m_{H}^{2}\right.$ in the SM)

$\delta m_{H}^{2}=c_{H} \lambda_{H S} m_{S}^{2} \log \frac{m_{S}^{2}}{Q^{2}}$

as follows from (1) (whose the quadratic part goes into curvature terms via symmergence [7]). It is clear that larger the $m_{S}$ larger the $\delta m_{H}^{2}$ and stronger the destabilization of the electroweak scale. The question is clear: How to prevent this destabilization of the electroweak scale? This is a profound question. And its answer is both obvious and obscure. It is obvious in that $\left|\lambda_{H S}\right|$ must be just small (as in (7) above) to start with since loop corrections to $\lambda_{H S}$ are proportional to $\lambda_{H S}$ itself (it remains small if it is small). It is, on the other hand, obscure in that there is no obvious selection rule or symmetry that can ensure the requisite smallness [32-35]. Its dimensionless nature disfavors also dynamical mechanisms like Giudice-Masiero mechanism [36] because a change like $\lambda_{H S} \rightarrow H^{\dagger} H / S^{2}$ would simply mean killing the coupling between $H$ and $S$. In the face of this impasse, a reasonable way to follow would be imposition of a judicious relationship among model parameters, with stabilization under the renormalization group flow. Indeed, symmergence, which sets $\lambda_{H S}$ free (as opposed to the known completions which require $\lambda_{H S}$ to remain close to the Higgs quartic coupling $\lambda_{H}$ as in (5) above), opens room for a mechanism in which $\lambda_{H S}$ can be linked to other model parameters in a way that keeps $\delta m_{H}^{2}$ under control. In this respect, the seesawic coupling in (7), which takes the particular form

$\lambda_{H S} \propto \lambda_{H} \frac{m_{H}^{2}}{m_{S}^{2}}$

for a BSM scalar $S$, possesses the right structure to keep $\delta m_{H}^{2}$ in (10) below $m_{H}^{2}$. It is clear that, in view of the bound (7), this coupling corresponds to the largest allowed values in that perfectly allowed smaller values result in feebler signals which are hard to detect.

The seesawic structure in (11) relates $\lambda_{H S}$ to the field masses. This means that for stabilizing the Higgs mass (suppressing $\delta m_{H}^{2}$ in (10)) the parameters in the potential must somehow enjoy a mass-dependent relationship beyond the energy conditions in (9). To this end, taking into account the perturbativity, we introduce a Mass-Degeneracy-Driven Unification (MDDU) linkup of the form (at a given scale $\left.Q=Q_{0}\right)$

$\lim _{m_{H}\left(Q_{0}\right) \rightarrow m_{S}\left(Q_{0}\right)} \lambda_{H}\left(Q_{0}\right)=\lambda_{S}\left(Q_{0}\right)=\left|\lambda_{H S}\left(Q_{0}\right)\right|$

which proves useful as it possesses the particular solution

$\lambda_{S}\left(Q_{0}\right)=\lambda_{H}\left(Q_{0}\right), \quad \lambda_{H S}\left(Q_{0}\right)=\frac{2 \lambda_{H}\left(Q_{0}\right)}{\frac{m_{H}^{2}\left(Q_{0}\right)}{m_{S}^{2}\left(Q_{0}\right)}+\frac{m_{S}^{2}\left(Q_{0}\right)}{m_{H}^{2}\left(Q_{0}\right)}}$

according to which $\lambda_{H S}\left(Q_{0}\right)$ reduces to the seesawic structure in (11) for $m_{S}\left(Q_{0}\right) \gg m_{H}\left(Q_{0}\right)$, and smoothly covers the opposite limit of $m_{S}\left(Q_{0}\right) \ll m_{H}\left(Q_{0}\right)$. It is clear the MDDU linkup (12) introduces a mass-dependent correlation among the quartic couplings in (8). It is special point in the parameter space (rather than a symmetry principle [32-35]). The particular MDDU scheme in (13) can be generalized to other portals in (2) by simply replacing $m_{S}^{2}$ with $M_{B S M}^{2}$, where $M_{B S M}=M_{Z^{\prime}}$ or $M_{B S M}=M_{N}$. This replacement rule can be useful for analyzing more general SM-BSM interactions.

It is only with UV completions like symmergence that it $\lambda_{h-B S M}$ gets loosened from the SM couplings (in view of the fixture in (5)), and it is with the MDDU in (12) that seesawic couplings like (7) or (11) become possible.

The changes in the MDDU linkup in (12) and (13) due to RGE flow of the parameters are analyzed in detail in the recent paper [37]. It is shown there that within the perturbative regime the MDDU relation is quite robust. Indeed, $\lambda_{H S}(Q)$ is small, it remains small as ensured by its RGE, and the $\lambda_{S}(Q)$ and $\lambda_{H}(Q)$ in turn remain essentially unchanged. Furthermore, mass of Higgs boson remains unaffected by the heavy scalar $S$ not at a specific scale $Q_{0}$ but at all scales from electroweak one to Planck one thanks to the smallness of $\lambda_{H S}(Q)$, as ensured the MDDU linkup. This shows that the MDDU works to ensure stability of the electroweak scale.

\section{The model}

In this section we analyze effects of an SM-singlet real scalar $S$ on the electroweak stability. It certainly is possible to consider a wider BSM sector and include all three types of the portals in (2). Nevertheless, the Higgs portal suffices for demonstrating the stability of the electroweak scale under the seesawic coupling in (13).

In view of the question (3), the most general, renormalizable, symmetric Lagrangian density extending the SM with a real singlet scalar field $S$ is given by $[38,39]$

$$
\mathcal{L}_{H S}=\left(D_{\mu} H\right)^{\dagger} D^{\mu} H+\frac{1}{2} \partial_{\mu} S \partial^{\mu} S-V_{H S},
$$


where $V(H, S)$ is the potential energy density in (8), and $H$ is the usual SM Higgs doublet

$H=\frac{1}{\sqrt{2}}\left(\begin{array}{c}\phi_{1}+i \phi_{2} \\ v_{H}+h+i \phi_{0}\end{array}\right)$.

with the Higgs boson $h$ remaining as a CP-even scalar after the Goldstone bosons $\phi_{i}$ are swallowed as longitudinal components of the $W$ and $Z$ bosons. Indeed, for $\lambda_{H}>0$ and $\lambda_{S}>0$, the potential gets bounded from below and the minimum of the potential breaks the electroweak symmetry spontaneously via the Higgs vacuum expectation value (VEV) $v_{H} \neq 0$. If the scalar $S$ is not inert (see for instance $[40,41])$, that is, if it gets a VEV $v_{S} \neq 0$ then the minimum of the potential (8) occurs at

$v_{H}^{2}=\frac{4 \lambda_{S} m_{H}^{2}-2 \lambda_{H S} m_{S}^{2}}{\lambda_{H S}^{2}-4 \lambda_{H} \lambda_{S}}, \quad v_{S}^{2}=\frac{4 \lambda_{H} m_{S}^{2}-2 \lambda_{H S} m_{H}^{2}}{\lambda_{H S}^{2}-4 \lambda_{H} \lambda_{S}}$

with the singlet boson $s$ defined as $S=v_{S}+s$ in parallel to (15).

In the vicinity of the vacuum (16), the mass-squared matrix of the $h$ and $s$ bosons

$M^{2}=\left(\begin{array}{cc}2 \lambda_{H} v_{H}^{2} & \frac{1}{2} \lambda_{H S} v_{H} v_{S} \\ \frac{1}{2} \lambda_{H S} v_{H} v_{S} & 2 \lambda_{S} v_{S}^{2}\end{array}\right)$

assume two eigenvalues

$m_{h_{1}}^{2}=\lambda_{H} v_{H}^{2}+\lambda_{S} v_{S}^{2}-\sqrt{\left(\lambda_{S} v_{S}^{2}-\lambda_{H} v_{H}^{2}\right)^{2}+\frac{1}{4} \lambda_{H S}^{2} v_{S}^{2} v_{H}^{2}}$,
$m_{h_{2}}^{2}=\lambda_{H} v_{H}^{2}+\lambda_{S} v_{S}^{2}+\sqrt{\left(\lambda_{S} v_{S}^{2}-\lambda_{H} v_{H}^{2}\right)^{2}+\frac{1}{4} \lambda_{H S}^{2} v_{S}^{2} v_{H}^{2}}$.

corresponding to the two physical eigenstates $h_{1}$ (which should be identified with the scalar boson observed at the LHC [1]) and $h_{2}$ (the extra scalar boson under search at the LHC and to be searched for at future colliders like the FCC). The key parameter is their mixing angle

$\tan 2 \theta=\frac{\lambda_{H S} v_{S} v_{H}}{\lambda_{S} v_{S}^{2}-\lambda_{H} v_{H}^{2}}$

which is proportional to $\lambda_{H S}-$ the strength of the SM-BSM coupling.

\section{One-loop corrections and model space}

In this section, we give a detailed analysis of the logarithmic corrections mentioned in (1). The Feynman diagrams which contributes the logarithmic corrections are depicted in Fig. 1. Leaving aside the quadratic corrections $c_{h} \Lambda^{2}$ in view of the

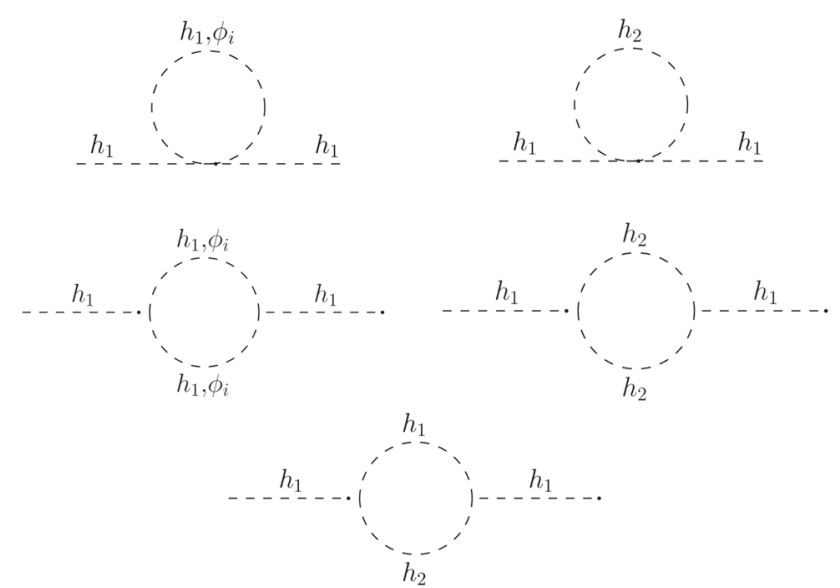

Fig. 1 The one-loop diagrams leading to the $m_{h_{1}}^{2}$ corrections in (1)

symmergence mechanism mentioned in the Introduction, we keep only the logarithmic corrections $\left(\Lambda \gg m_{h_{2}} \gg m_{h_{1}}\right)$

$$
\begin{aligned}
\left(\delta m_{h_{1}}^{2}\right)_{l o g}= & \frac{1}{8 \pi^{2}}\left[\left(6 \lambda_{h_{1} h_{1} h_{1} h_{1}}+3 \lambda_{h_{1} h_{1} \phi \phi}\right) m_{h_{1}}^{2}\right. \\
& \left.+\left(9 \lambda_{h_{1} h_{1} h_{1}}^{2}+3 \lambda_{h_{1} \phi \phi}^{2}\right)\right] \log \left(\frac{m_{h_{1}}^{2}}{\Lambda^{2}}\right) \\
& +\frac{1}{16 \pi^{2}}\left[2 \lambda_{h_{1} h_{1} h_{2} h_{2}} m_{h_{2}}^{2}+2 \lambda_{h_{1} h_{2} h_{2}}^{2}\right. \\
& \left.+\lambda_{h_{1} h_{1} h_{2}}^{2}\right] \log \left(\frac{m_{h_{2}}^{2}}{\Lambda^{2}}\right)
\end{aligned}
$$

where the various couplings (like the quartic couplings $\left.\lambda_{h_{i}} h_{j} h_{k} h_{l}\right)$ are listed explicitly in the Appendix as functions of $\lambda_{H}, \lambda_{S}, \lambda_{H S}$ and the mixing angle $\theta$.

The $h_{1}$ mass receives non-trivial corrections from the $h_{2}$ loops. This feature, explicated in (20), requires $\lambda_{H S}$ to be bounded appropriately. The vacuum stability already gives a bound (as follows from (9))

$\lambda_{H S}^{2} \leq 16 \lambda_{H} \lambda_{S}$

which means that $\left|\lambda_{H S}\right|$ is typically at the $30 \%$ level depending on precise values of $\lambda_{H}$ and $\lambda_{S}$. We will consider different parameter ranges during the analysis.

The bound above is however not sufficient to ensure electroweak stability. The reason is that $h_{2}$ can be too heavy to keep $h_{1}$ mass within the LHC bound. To this end, one comes back to the see-sawic bound in (7). In what follows thus we require thus $\lambda_{H S}$ to have the value

$\lambda_{H S}=\frac{m_{H}^{2}}{m_{S}^{2}}$

after expressing

$m_{H}^{2}=\frac{1}{4 v_{H}^{2}}\left(2 \lambda_{S} v_{S}^{4}-4 \lambda_{H} v_{H}^{4}-v_{S}^{4}\right.$ 


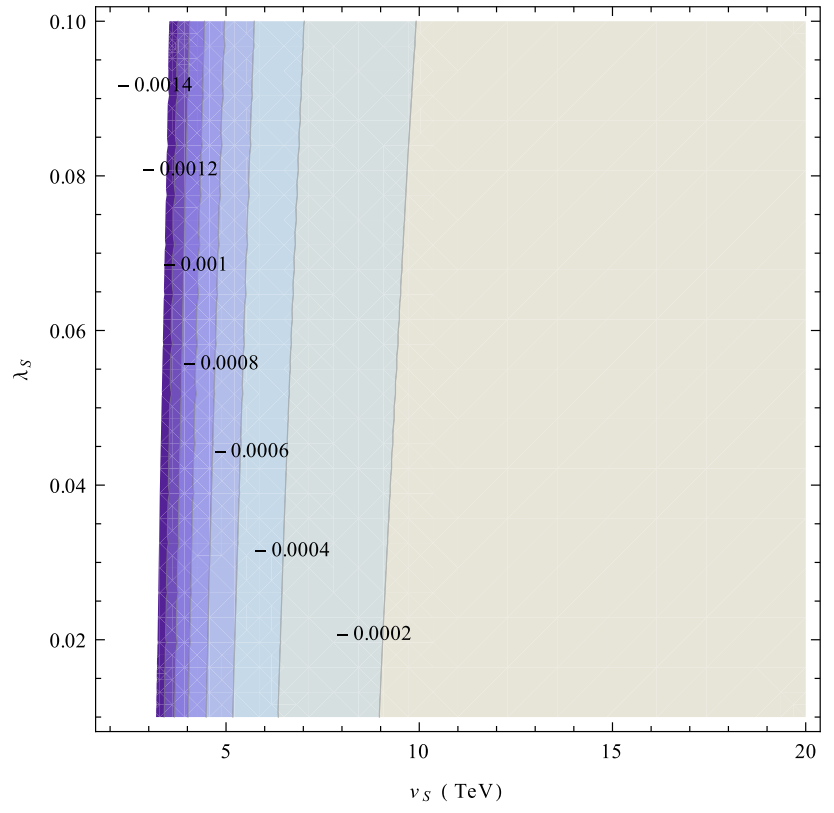

Fig. 2 Variation of $\lambda_{H S}$ with $v_{S}(\mathrm{TeV})$ and $\lambda_{S}$ for $0.01 \leq \lambda_{S} \leq 0.1$

$$
\begin{aligned}
& \left.+\sqrt{8 \lambda_{H} v_{H}^{4} v_{S}^{4}+v_{S}^{8}-4 \lambda_{S} v_{S}^{8}+4 \lambda_{S}^{2} v_{S}^{8}}\right), \\
m_{S}^{2}= & \frac{1}{4}\left(-\left(1+2 \lambda_{S}\right) v_{S}^{2}\right. \\
& \left.+\sqrt{8 \lambda_{H} v_{H}^{4}+v_{S}^{4}-4 \lambda_{S} v_{S}^{4}+4 \lambda_{S}^{2} v_{S}^{4}}\right) .
\end{aligned}
$$

as functions of the $H$ and $S$ VEVs. Trading two model parameters for the VEVs in this form leads us to the physical shell set by the VEVs. In fact, we hereon specialize to the LHC values

$\lambda_{H}=0.13, \quad v_{H}=246.2 \mathrm{GeV}$

and analyze the model in terms of the remaining two free parameters: the $S$ quartic coupling $\lambda_{S}$ and the $S$ VEV $v_{S}$.

The allowed ranges of the model parameters can be determined numerically. In doing so we consider $v_{S}$ values as large as $20 \mathrm{TeV}$ in view of the sensitivity of the exotica searches at the LHC [3]. To this end, we plot in Fig. 2 variation of $\lambda_{H S}$ with $v_{S}$ in the small $\lambda_{S}$ regime of $0.01 \leq \lambda_{S} \leq 0.1$. It is seen that $\lambda_{H S}$, which decreases with $m_{S}^{2}$ due to its see-sawic structure in (22), in magnitude, remains below $\lambda_{S}$ at least by two orders of magnitude.

Shown in Fig. 3 is the variation of $\lambda_{H S}$ with $v_{S}$ in the large $\lambda_{S}$ regime of $0.1 \leq \lambda_{S} \leq 0.5$. It is clear that $\lambda_{H S}$, in magnitude, remains below $\lambda_{S}$ at least by two orders of magnitude.

For larger $\lambda_{S}$, from 0.5 to 0.9 , we find that $\lambda_{H S}$ takes unacceptably large values (a thousand), we do not consider therefore $\lambda_{S}$ values above 0.5 . In fact, hereon we set $\lambda_{S}=0.1$ as a nominal value revealing the physics implications of the heavy scalar.

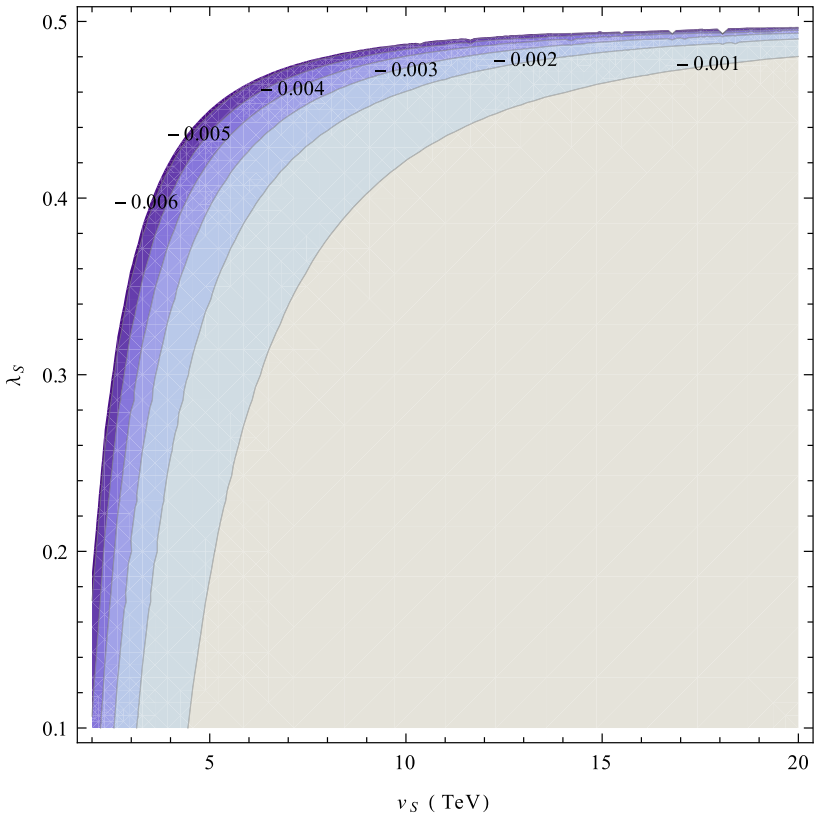

Fig. 3 Variation of $\lambda_{H S}$ with $v_{S}(\mathrm{TeV})$ and $\lambda_{S}$ for $0.1 \leq \lambda_{S} \leq 0.5$

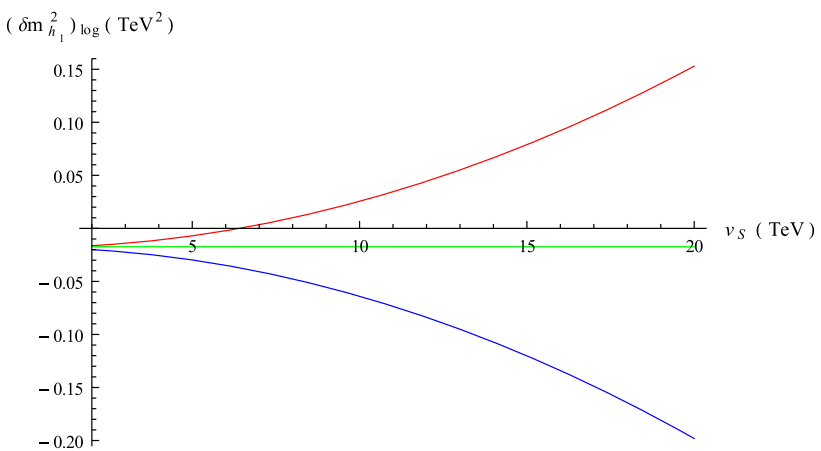

Fig. 4 Corrections to the Higgs mass as a function of $v_{S}$ for $\lambda_{H S}=$ -0.01 (red), $\lambda_{H S}=0.01$ (blue) and $\lambda_{H S}=m_{H}^{2} / m_{S}^{2}$ (green)

To see the difference between setting $\lambda_{H S}$ to a fixed (albeit small) value as in most phenomenological analyses [40] and requiring $\lambda_{H S}$ to obey the see-sawic bound in (22) we plot in Fig. $4 \delta m_{h_{1}}^{2}$ in TeV as a function of $v_{S}$. It is clear that the seesawic structure provides us with a rather stable electroweak scale.

To see further how $\lambda_{H S}$ varies with $v_{S}$ we list in Table 1 $\lambda_{H S}$ values as $v_{S}$ ranges from 2 to $20 \mathrm{TeV}$. In agreement with Figs. 2 and $3, \lambda_{H S}$ remains negative throughout and well satisfies the vacuum stability bound (21). It is clear that larger the $m_{S}$ of scalar field, the weaker its interaction with Higgs. This decrease could explain why we have not observed any fingerprint of BSM physics (the scalar $S$ here) at LHC experiments.

Before closing this section, it is worth noting that any value of $\lambda_{H S}$ obeying the constraint (7) can satisfy the electroweak stability. However, it is not difficult to see that smaller $\lambda_{H S}$ 
Table 1 The changes in the parameter $\lambda_{H S}$ as $v_{S}\left(m_{h_{2}}\right)$ increases

\begin{tabular}{lll}
\hline$v_{S}(\mathrm{GeV})$ & $m_{h_{2}}(\mathrm{GeV})$ & $\lambda_{H S}$ \\
\hline 2000 & 894.428 & $-4.9 \times 10^{-3}$ \\
3000 & 1341.64 & $-2.2 \times 10^{-3}$ \\
4000 & 1788.85 & $-1.2 \times 10^{-3}$ \\
5000 & 2236.07 & $-8.0 \times 10^{-4}$ \\
6000 & 2683.28 & $-5.5 \times 10^{-4}$ \\
7000 & 3130.5 & $-4.0 \times 10^{-4}$ \\
8000 & 3577.71 & $-3.0 \times 10^{-4}$ \\
9000 & 4024.92 & $-2.4 \times 10^{-4}$ \\
10,000 & 4472.14 & $-2.0 \times 10^{-4}$ \\
15,000 & 6708.2 & $-8.7 \times 10^{-5}$ \\
20,000 & 8944.27 & $-4.9 \times 10^{-5}$ \\
\hline
\end{tabular}

$\left(<m_{H}^{2} / m_{S}^{2}\right.$ ) leads to the requirement of larger luminosities and energies for the discovery of new physics at colliders. The maximal value of $\lambda_{H S}\left(=m_{H}^{2} / m_{S}^{2}\right)$ provides one with accessible and realistic luminosities and energies for discovery. This is the reason why we have considered $\lambda_{H S}$ in (22) throughout our analyses.

\section{Collider phenomenology}

In this section we perform a detailed simulation study to answer the question (3) in the Introduction. The analysis involves production and decay rates as well as event selection and background analysis. Below is a systematic discussion of the analysis stages.

The production cross section of real singlet scalar depends on its mass and its coupling to the SM Higgs field. In view of the see-sawic coupling (22) the production cross section is directly set by $m_{h_{2}}$ (or $v_{S}$ ). It sets also branching fractions of $h_{2}$ decays. The branching fractions of $h_{2}$ into various SM particles are given in Fig. 5. The dominant decay channels are seen to be $h_{2} \rightarrow W W(49 \%), h_{2} \rightarrow h_{1} h_{1}(25 \%)$ and $h_{2} \rightarrow Z Z(24 \%)$, which are almost independent of $m_{h_{2}}$. This constancy of the branching fractions, a property following from the seesawic couplings in (11) and (13), proves useful for putting discovery limits (as in the simplified models [42]).

In Fig. $6, h_{2}$ production cross sections times branching ratios are given as a function of $m_{h_{2}}$ for the $p p \rightarrow h_{2} \rightarrow$ $W^{+} W^{-} \rightarrow l v j j, p p \rightarrow h_{2} \rightarrow W^{+} W^{-} \rightarrow l^{+} v l^{-} v$, $p p \rightarrow h_{2} \rightarrow Z Z \rightarrow l^{+} l^{-} j j$ and $p p \rightarrow h_{2} \rightarrow Z Z \rightarrow 4 l$ channels separately for $\sqrt{s}=14 \mathrm{TeV}$ and $\sqrt{s}=100 \mathrm{TeV}$. Although $p p \rightarrow h_{2} \rightarrow W^{+} W^{-} \rightarrow l v j j$ channel has the highest cross section, reconstruction of this type of semileptonic final states i.e. a charged lepton (electron or muon) is challenging due to the large missing transverse momentum coming from the presence of a neutrino in each event and at

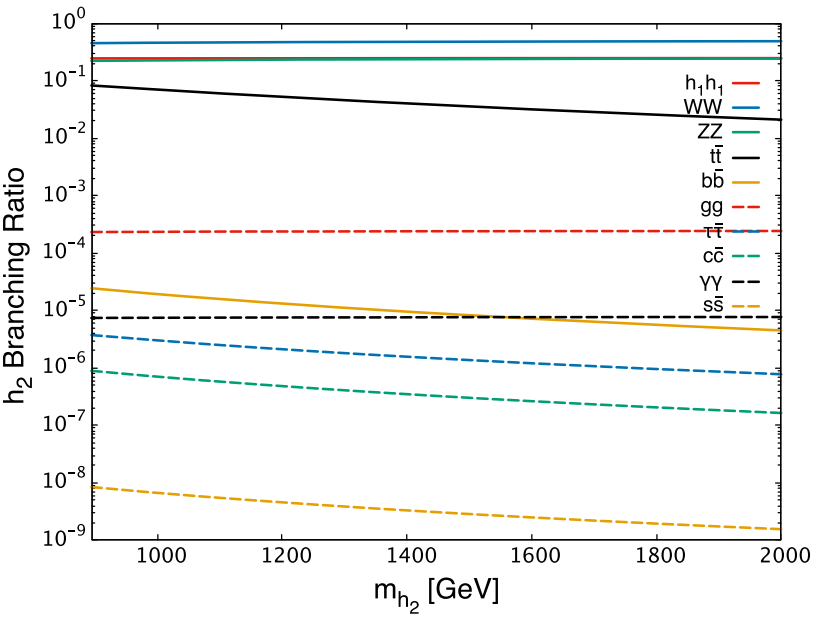

Fig. 5 Branchings of the heavy scalar $h_{2}$ into various SM particles. The $h_{1} h_{1}$, WW and ZZ are the dominant decay modes. The decay rates remain constant essentially or decrease with $m_{h_{2}}$ due to the see-sawic coupling

least two jets. In addition, W+jets background is dominant and gives a peak in the same region with diboson invariant mass, making it extremely difficult to separate signal from the background [43]. The $p p \rightarrow h_{2} \rightarrow W^{+} W^{-} \rightarrow l^{+} v l^{-} v$ channel, which has the second highest cross section, is another challenging channel since the invariant mass of the system is not completely reconstructable due to the missing energy in the final states coming from the neutrinos [44].

In $p p \rightarrow h_{1} h_{1}$ channel, it is possible to search for $h_{2}$ via $4 \gamma$ (suppressed by loops), $\gamma \gamma b \bar{b}$ (suppressed by loops and small bottom Yukawa), and the like. We will not take into account these channels in our analysis.

The $p p \rightarrow h_{2} \rightarrow Z Z \rightarrow l^{+} l^{-} j j$ channel has a higher cross section than $p p \rightarrow h_{2} \rightarrow Z Z \rightarrow 4 l$ and therefore may bring more statistics, but due to the jets in the final states, this channel has larger background especially from $\mathrm{Z}+\mathrm{jets}$ and $t \bar{t}$ processes. The $p p \rightarrow h_{2} \rightarrow Z Z \rightarrow 4 l$ has smaller cross sections than the other channels presented in Fig. 6, but it has completely reconstructable final state (four charged leptons) and has lower background contamination $[45,46]$. Therefore, we focus on the $4 l$ channel and perform a detailed analysis using various different mass scenarios.

In total, we consider seven different mass scenarios between $800-2000 \mathrm{GeV}$ in increments of $200 \mathrm{GeV}$, for the search of $h_{2}$ via the $4 l$ decay channel. We present kinematic distributions and the event selection efficiencies for the low mass and high mass scenarios, $m_{h_{2}}=800 \mathrm{GeV}$ and $m_{h_{2}}=2000 \mathrm{GeV}$. The final results are given for every mass value considered in the analysis. All analyses are carried out at two different center of mass energies; at $\sqrt{s}=14 \mathrm{TeV}$ and $\sqrt{s}=100 \mathrm{TeV}$, corresponding, respectively, to the center of mass energy of the $p p$ collisions at the high luminosity 

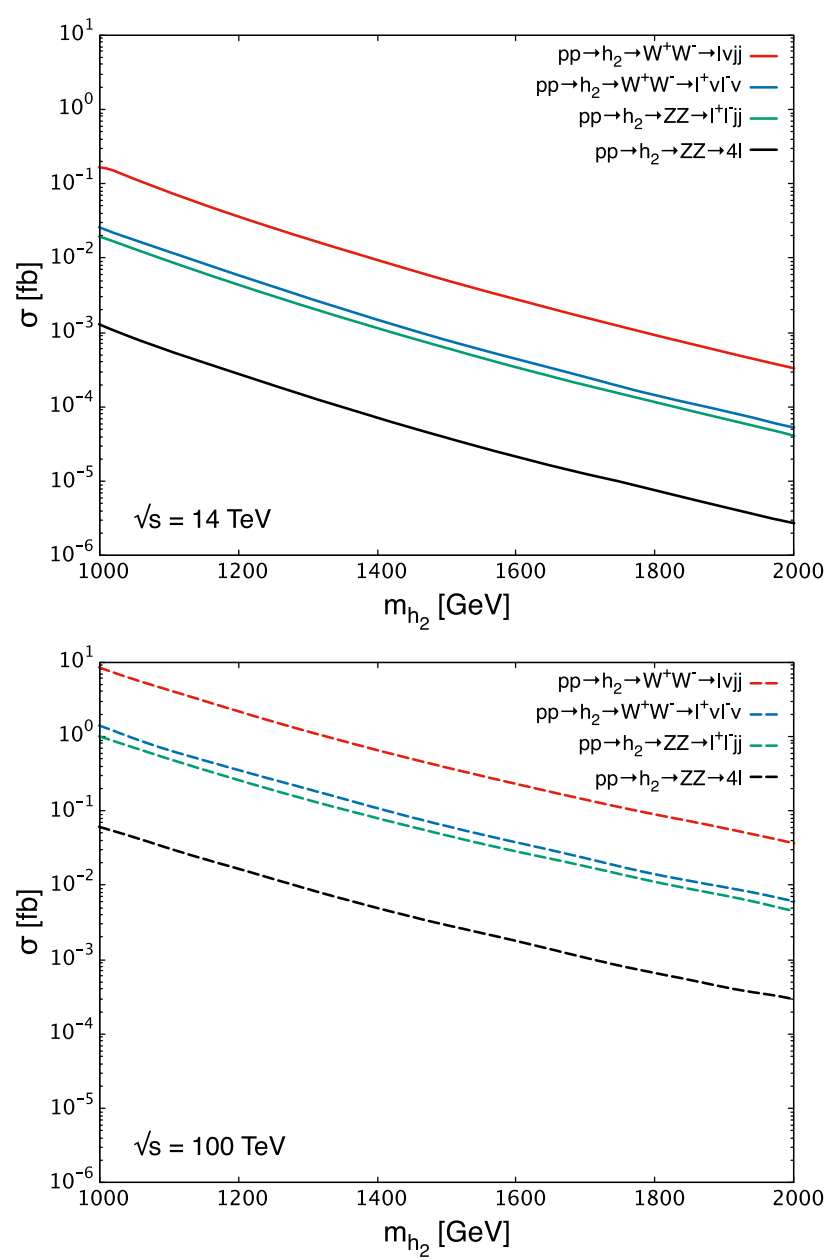

Fig. 6 Cross section (fb) times branching ratio as functions of $m_{h_{2}}$ for the channels $p p \rightarrow h_{2} \rightarrow W^{+} W^{-} \rightarrow l v j j$ (red line), $p p \rightarrow h_{2} \rightarrow$ $W^{+} W^{-} \rightarrow l^{+} v l^{-} v$ (blue line), $p p \rightarrow h_{2} \rightarrow Z Z \rightarrow l^{+} l^{-} j j$ (green line) and $p p \rightarrow h_{2} \rightarrow Z Z \rightarrow 4 l$ (black line) for $\sqrt{s}=14 \mathrm{TeV}$ (up) and $\sqrt{s}=100 \mathrm{TeV}$ (down)

phase of the LHC (HL-LHC) and the future circular collider FCC-hh [47-49].

In simulating the $4 l$ signal and the SM background events for $p p \rightarrow Z Z$, whose Feynman diagrams are depicted in Fig. 7, we have modified the SM package in LanHEP v3.2.0 [50] by including the real singlet $S$, and exported the extended model to CalcHEP v3.7.5 [51]. The events are simulated by CalcHEP using the LHAPDF v6.1.6 [52] library and its CTEQ6L1 [53] parton distribution functions (PDFs) as well as PYTHIA8 v2.3.0 [54] for parton showering and hadronization. The detector response is simulated by Delphes v3.4.2 fast-simulation package [55] using the HL-LHC and FCC-hh detector card files implemented in it. Events are analyzed using the ExRootAnalysis package linked to ROOT v6.12 [56].

To search for the heavy scalar $h_{2}$ in $p p \rightarrow h_{2} \rightarrow Z Z \rightarrow$ $4 l$ channel, we select events with four leptons in the final state,

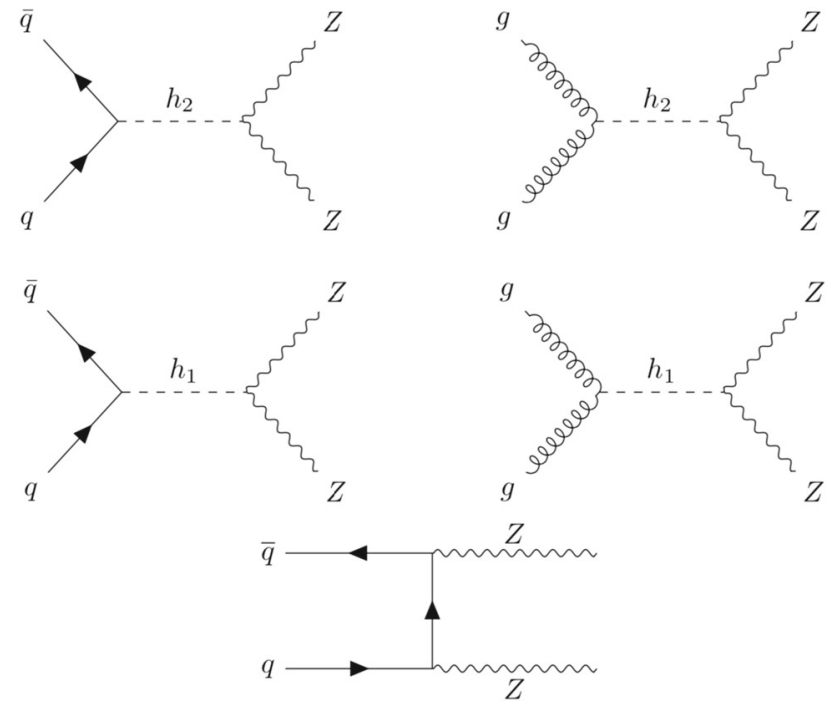

Fig. 7 The leading order Feynman diagrams for the signal process (a) and the main background process (b)

Table 2 Pre-selection cuts for identifying the leptons $(l=e$ or $\mu)$

\begin{tabular}{ll}
\hline Quantity & Selection criteria \\
\hline Transverse momentum & $p_{\mathrm{T}}^{l}>10 \mathrm{GeV}$ \\
Pseudo-rapidity & $\left|\eta_{l}\right|<2.5$ \\
Radial distance & $\Delta \mathrm{R}\left(l_{i}, l_{j}\right)>0.2$ \\
\hline
\end{tabular}

where each lepton is either an electron or a muon. The two $\mathrm{Z}$ bosons in the events, each decaying to opposite-sign, sameflavor (electrons or muons) lepton pair, lead to three possible final states configurations; $4 e, 4 \mu$, and $2 e 2 \mu$. In each selected event, two $\mathrm{Z}$ boson candidates are reconstructed from lepton pairs considering all possible pairing combinations and the one with the mass closer to the mass of the $\mathrm{Z}$ boson is called $Z_{1}$ and the other reconstructed $\mathrm{Z}$ boson is $Z_{2}$. Following a similar analysis strategy and event selection as in $[57,58]$, we applied the pre-selection cuts listed in Table 2. In this table, $\Delta \mathrm{R}$ is the distance between two leptons in the $\eta-\phi$ plane and defined as $\Delta \mathrm{R}\left(l_{i}, l_{j}\right)=\sqrt{\left(\eta_{i}-\eta_{j}\right)^{2}-\left(\phi_{i}-\phi j\right)^{2}}$ where $i$ and $j$ refer to the lepton pairs used in reconstruction of the $\mathrm{Z}$ bosons.

Depicted in Figs. 8, 9, 10, 11 and 12 are the various kinematic distributions subjected to the pre-selection cuts in Table 2. Depicted in Fig. 8, on the other hand, are the transverse momentum $\left(p_{T}\right)$ spectra of the leading, secondleading, third-leading, and fourth-leading lepton in signal events for $m_{h_{2}}=800 \mathrm{GeV}$ and $m_{h_{2}}=2000 \mathrm{GeV}$ at $\sqrt{s}=14 \mathrm{TeV}$. The background contribution is also presented. As shown in figure, the leptons originating from $\mathrm{Z}$ boson decays in signal events (note that $\mathrm{Z}$ is the decay product of $m_{h_{2}}$ ) have harder $p_{T}$ spectrum while the leptons in background events significantly dominate in lower transverse 

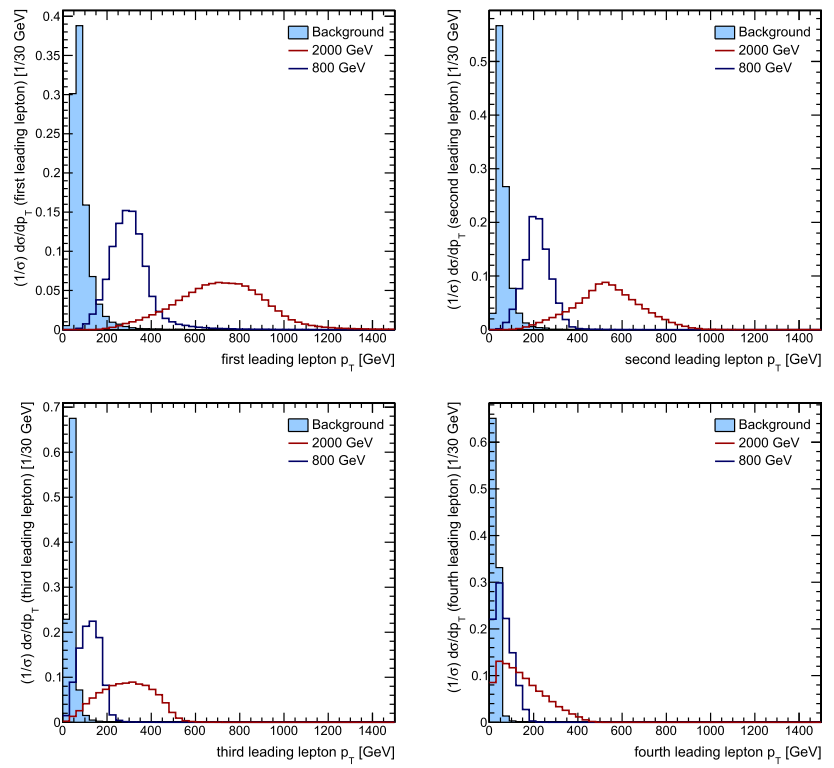

Fig. 8 Normalized differential cross sections as a function of leading lepton $p_{T}(\mathbf{a})$, second-leading lepton $p_{T}(\mathbf{b})$, third-leading lepton $p_{T}$ (c) and fourth-leading lepton $p_{T}$ (d) for $m_{h_{2}}=2000 \mathrm{GeV}$ (red line), $800 \mathrm{GeV}$ (blue line) and background (blue solid) at $\sqrt{s}=14 \mathrm{TeV}$

momenta. The same distributions are given in Fig. 10 for the events at $100 \mathrm{TeV}$ and the conclusion is the same: leptons in signal events have harder $p_{T}$ spectra than the leptons in the background events. This feature makes lepton $p_{T}$ a decisive discriminator for extracting the signal from the background.

Given in Fig. 9 are the transverse momenta of the $\mathrm{Z}_{1}$ and $\mathrm{Z}_{2}$ bosons ( $p_{T_{Z 1}}$ and $\left.p_{T_{Z 2}}\right)$ as well as the invariant mass $\left(m_{4 l}\right)$ and transverse momentum $\left(p_{T_{4 l}}\right)$ of the four-lepton system. The background is represented by the blue histograms. The same distributions are shown in Fig. 11 for $\sqrt{s}=100 \mathrm{TeV}$. In similarity to the lepton $p_{T}$ spectra, the $p_{T}$ distributions of $Z_{1}$ and $Z_{2}$ bosons in the signal events, too, are seen to have higher transverse momentum compared to those in the background.

It is clear from Figs. 9 and 11 that a cut of $p_{T}>120 \mathrm{GeV}$ on the transverse momenta of the both $\mathrm{Z}_{1}$ and $\mathrm{Z}_{2}$ bosons suppresses significant portion of background events. Thus, it is possible to use also these distributions to distinguish the signal from the background. Moreover, the invariant mass of the four lepton system $\left(m_{4 l}\right)$ turns out to be one of the most sensitive observables in that it gives a narrow peak around the actual $m_{h_{2}}$ value, and it can thus be used to extract the signal especially when $m_{h_{2}}$ is large (for example, much larger than the $2 m_{\mathrm{Z}}$ ). (We do not use $p_{T}$ of the four-lepton system in event selection but we give them in Figs. 9d and 11d, for completeness.)

The Z-boson mass is a good measure of the success of the signal extraction. Shown in Fig. 12 is the reconstruction of the two $\mathrm{Z}$ boson candidates in the signal and background events
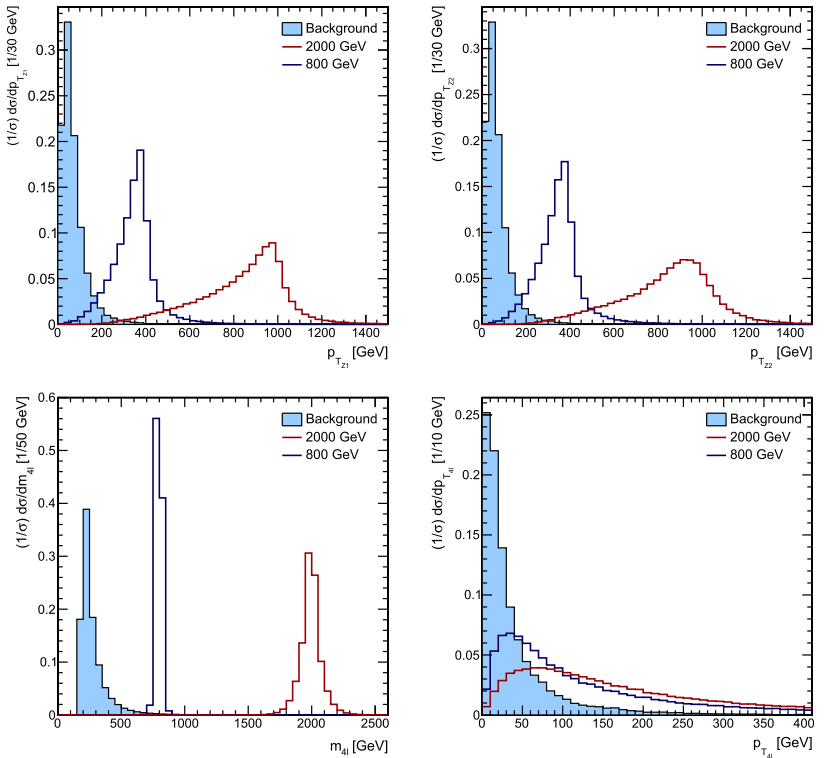

Fig. 9 Normalized differential cross sections as a function of the transverse momentum of $\mathrm{Z}_{1}$ boson (a) $\mathrm{Z}_{2}$ boson (b), and the invariant mass $(\mathbf{c})$ and transverse momentum (d) of the four-lepton system for $m_{h_{2}}=2000 \mathrm{GeV}$ (red line), $800 \mathrm{GeV}$ (blue line) and background (blue solid) at $\sqrt{s}=14 \mathrm{TeV}$
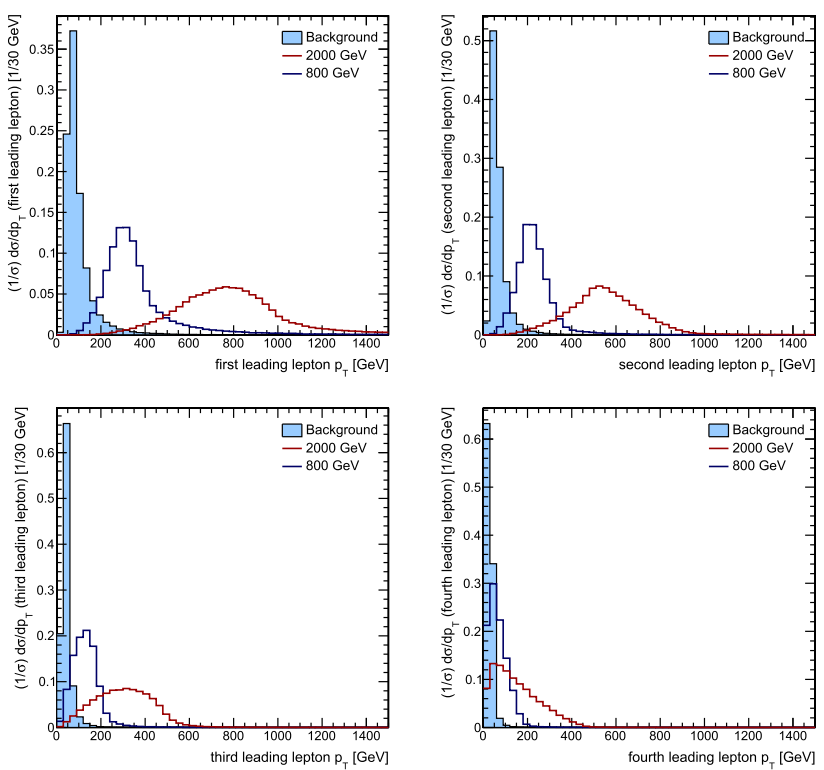

Fig. 10 Normalized differential cross sections as a function of leading lepton $p_{T}$ (a), second-leading lepton $p_{T}$ (b), third-leading lepton $p_{T}$ (c) and fourth-leading lepton $p_{T}$ (d) for $m_{h_{2}}=2000 \mathrm{GeV}$ (red line), $800 \mathrm{GeV}$ (blue line) and background (blue solid) at $\sqrt{s}=100 \mathrm{TeV}$

at 14 and $100 \mathrm{TeV}$. This figure shows the invariant mass of the reconstructed $\mathrm{Z}$ candidates and $\Delta \mathrm{R}\left(\mathrm{Z}_{1}, \mathrm{Z}_{2}\right)$ distribution between the $Z$ pairs. The reconstructed $Z$ boson mass is seen to sharply peaked at the actual Z-mass $M_{Z} \sim 91.19 \mathrm{GeV}$, ensuring that $\mathrm{Z}$ pairs in the events are reconstructed accu- 

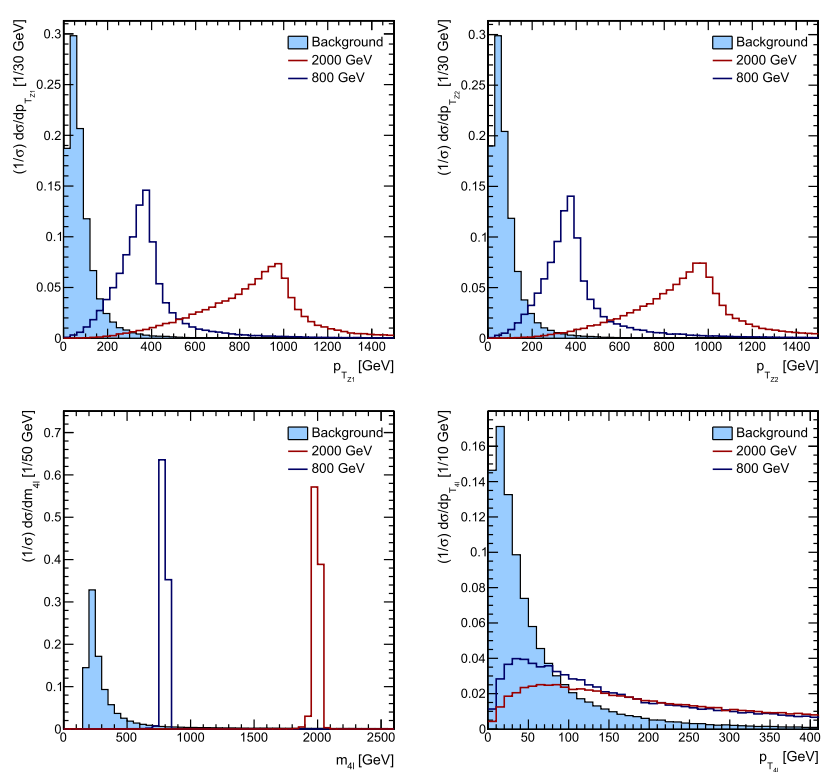

Fig. 11 Normalized differential cross sections as a function of the transverse momentum of $\mathrm{Z}_{1}$ boson (a) $\mathrm{Z}_{2}$ boson (b), and the invariant mass (c) and transverse momentum (d) of the four-lepton system for $m_{h_{2}}=2000 \mathrm{GeV}$ (red line), $800 \mathrm{GeV}$ (blue line) and background (blue solid) at $\sqrt{s}=100 \mathrm{TeV}$
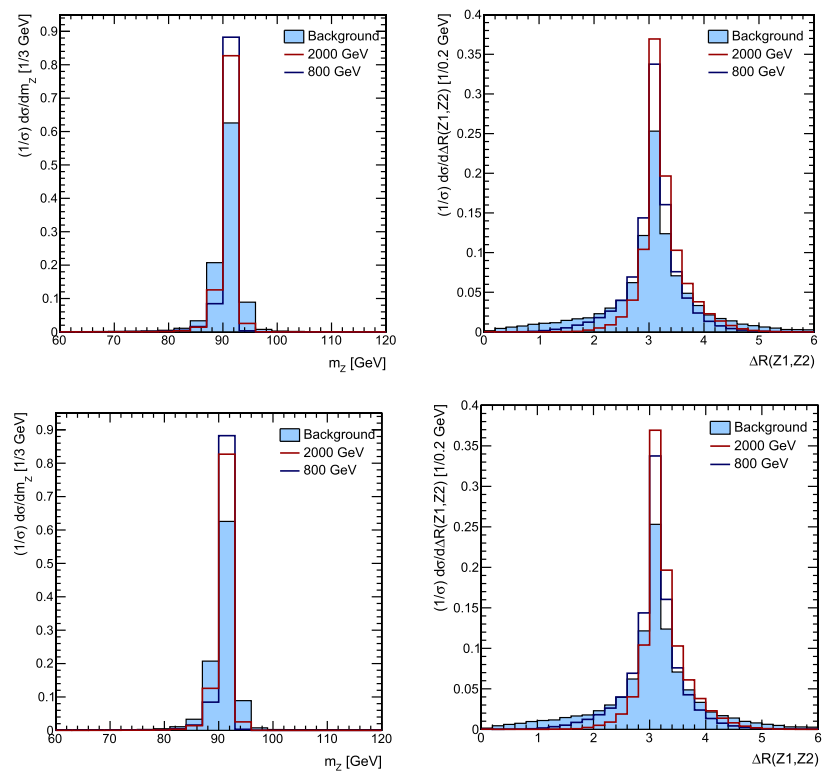

Fig. 12 Invariant mass of the reconstructed $\mathrm{Z}$ boson candidates in the events (left) and angular separation $\left(\Delta \mathrm{R}\left(\mathrm{Z}_{1}, \mathrm{Z}_{2}\right)\right)$ between the two reconstructed $Z$ bosons (right) at $14 \mathrm{TeV}$ (up) and $100 \mathrm{TeV}$ (down). The peak at $\sim 3$ in $\Delta \mathrm{R}\left(\mathrm{Z}_{1}, \mathrm{Z}_{2}\right)$ distributions both for signal and background show that the $\mathrm{Z}$ bosons are produced mostly back-to-back

rately enough. The peak at $\Delta \mathrm{R}\left(\mathrm{Z}_{1}, \mathrm{Z}_{2}\right) \simeq 3$ reveals that the two $\mathrm{Z}$ bosons are mostly produced back-to-back.

For discriminating the signal from the background we apply a second set of selection criteria. Given in Table 3 are the event selection and cumulative percentage efficien- cies after the sequential application of each cut, for the signal and background events at $14 \mathrm{TeV}$. The same data for $100 \mathrm{TeV}$ samples is listed in Table 4. In addition to Fig. 12, it can be seen also from these tables that the $\mathrm{Z}$ pairs in the events are reconstructed accurately enough. Despite the rather tight cut applied on the mass of the $\mathrm{Z}$ pairs $\left(\mathrm{Z}_{1}\right.$ and $\left.\mathrm{Z}_{2} \in \mathrm{Z} \pm 10 \mathrm{GeV}\right)$, only approximately $4-5 \%$ of the events are rejected, meaning that the $\mathrm{Z}$ bosons produced in the events are already within the $M_{\mathrm{Z}} \pm 10 \mathrm{GeV}$ mass range. A large portion of the background signal is eliminated by the cut applied over the lepton momenta. Approximately $90 \%$ of the background at $14 \mathrm{TeV}$ and approximately $80 \%$ at $100 \mathrm{TeV}$ are eliminated by the cuts applied on the transverse momentum of the leptons in the final states. The same cuts reject at most $15 \%$ of the signal events at $800 \mathrm{GeV}$ and $5 \%$ at $2000 \mathrm{GeV}$. The remaining background can be further suppressed with the additional cuts applied over the mass of the four lepton system $\left(m_{4 l}\right)$ and over the transverse momenta of the $Z_{1}$ and $\mathrm{Z}_{2}$ bosons. However, compared to the signal processes, high production cross sections of the processes contributing to the background cause significant number of background events to arise at high luminosities. All these properties can be read off from Table 3 (for $14 \mathrm{TeV}$ ) and Table 4 (for $100 \mathrm{TeV}$ ).

After performing all the event selections and taking into account the expected total integrated luminosity values for the HL-LHC and FCC-hh, we compute the signal and background event yields as well as the signal significancies. The significancy is defined as

$n=\frac{S}{\sqrt{S+B}}$

where $S(B)$ is the number of signal (background) events which passed all the selection cuts.

The results obtained are plotted in Fig. 13 for FCC-hh and HL-LHC as a function of the $h_{2}$ mass. The dots on the plot show the signal significance for the corresponding mass value and the red and blue solid lines show the linear fits applied to the FCC-hh and HL-LHC values, respectively. The red-dashed and red-dotted lines show, respectively, the upper limits on $m_{h_{2}}$ at $3 \sigma$ (observation) and $5 \sigma$ (discovery).

It is clear that the resulting signal significances for the HL-LHC are too low: $0.3 \sigma$ at $800 \mathrm{GeV}$ and gradually drops to $0.0001 \sigma$ at $2000 \mathrm{GeV}$. Therefore, it is clear that for the expected luminosity values of HL-LHC, which is $3 \mathrm{ab}^{-1}$, no significant excess of signal over the background is observed in the $800 \mathrm{GeV}<m_{h_{2}}<2000 \mathrm{GeV}$ mass range. The results are more promising for FCC-hh. The higher center-of-mass energy of the collisions as well as the larger expected total integrated luminosity $\left(100 \mathrm{TeV}, \mathrm{L}_{\text {int }}=20 \mathrm{ab}^{-1}\right)$, enhance the signal significances by a factor of about 100 . At the FCChh, $h_{2}$ can be discovered with a significance of $5 \sigma$ and a 
Table 3 Effects of various selection cuts for the analysis of signal and background events at $14 \mathrm{TeV}$. The values for the background are given for $m_{h_{2}}=800 \mathrm{GeV}$ by default while the values in parenthesis indicate the remaining percentage of events for $m_{h_{2}}=2000 \mathrm{GeV}$

\begin{tabular}{llll}
\hline Event selection & Background $(\%)$ & $m_{h_{2}}=800 \mathrm{GeV}(\%)$ & 100 \\
\hline All & 100 & & 100 \\
Triggered & & 32.6 & 36.8 \\
Number of pre-selected leptons $\geq 4$ & 16.3 & & 31.9 \\
$\mathbf{Z}$ reconstruction & & & 34.6 \\
Invariant mass of $m_{\mathrm{Z}_{1}}$ and $m_{\mathrm{Z}_{2}} \in m_{\mathrm{Z}} \pm 10 \mathrm{GeV} / \mathrm{c}^{2}$ & 15.7 & 31.9 & 34.6 \\
Signal selection & & 31.8 & 34.6 \\
Leading lepton $p_{T}>90 \mathrm{GeV} / \mathrm{c}$ & 4.6 & 29.8 & 34.3 \\
2nd leading lepton $p_{T}>70 \mathrm{GeV} / \mathrm{c}$ & 3.4 & 27.2 & 33.1 \\
3rd leading lepton $p_{T}>50 \mathrm{GeV} / \mathrm{c}$ & 1.8 & & 14.6 \\
4th leading lepton $p_{T}>20 \mathrm{GeV} / \mathrm{c}$ & 1.5 & 24.9 & 14.6 \\
Invariant mass of four lepton system & & 24.6 & \\
$\quad\left(m_{4 l}\right) \in m_{h_{2}} \pm 30 \mathrm{GeV} / \mathrm{c}^{2}$ & $0.03(0.0008)$ & \\
$\quad p_{T_{Z_{1}}}>120 \mathrm{GeV} / \mathrm{c}$ and $p_{T_{Z_{2}}}>120 \mathrm{GeV} / \mathrm{c}$ & $0.03(0.0008)$ & & \\
\hline
\end{tabular}

Table 4 Effects of various selection cuts for the analysis of signal and background events at $100 \mathrm{TeV}$. The values for the background are given for $m_{h_{2}}=800 \mathrm{GeV}$ by default while the values in parenthesis indicate the remaining percentage of events for $m_{h_{2}}=2000 \mathrm{GeV}$

\begin{tabular}{|c|c|c|c|}
\hline Event selection & Background (\%) & $m_{h_{2}}=800 \mathrm{GeV}(\%)$ & $m_{h_{2}}=2000 \mathrm{GeV}(\%)$ \\
\hline All & 100 & 100 & 100 \\
\hline \multicolumn{4}{|l|}{ Triggered } \\
\hline Number of pre-selected leptons $\geq 4$ & 7.8 & 34.9 & 44.3 \\
\hline \multicolumn{4}{|l|}{$\mathrm{Z}$ reconstruction } \\
\hline Invariant mass of $m_{\mathrm{Z}_{1}}$ and $m_{\mathrm{Z}_{2}} \in m_{\mathrm{Z}} \pm 10 \mathrm{GeV}^{2}$ & 7.4 & 34.3 & 43.9 \\
\hline \multicolumn{4}{|l|}{ Signal selection } \\
\hline Leading lepton $p_{T}>90 \mathrm{GeV} / \mathrm{c}$ & 3.3 & 34.2 & 43.9 \\
\hline 2nd leading lepton $p_{T}>70 \mathrm{GeV} / \mathrm{c}$ & 2.7 & 34.1 & 43.9 \\
\hline 3rd leading lepton $p_{T}>50 \mathrm{GeV} / \mathrm{c}$ & 1.9 & 32.2 & 43.6 \\
\hline 4th leading lepton $p_{T}>20 \mathrm{GeV} / \mathrm{c}$ & 1.7 & 29.5 & 43.1 \\
\hline \multicolumn{4}{|l|}{ Invariant mass of four lepton system } \\
\hline$\left(m_{4 l}\right) \in m_{h_{2}} \pm 30 \mathrm{GeV} / \mathrm{c}^{2}$ & $0.04(0.01)$ & 29.0 & 37.4 \\
\hline$p_{T_{Z_{1}}}>120 \mathrm{GeV} / \mathrm{c}$ and $p_{T_{Z_{2}}}>120 \mathrm{GeV} / \mathrm{c}$ & $0.04(0.01)$ & 28.4 & 37.4 \\
\hline
\end{tabular}

mass up to $\sim 870 \mathrm{GeV}$. The upper limit on the mass for $3 \sigma$ evidence is $\sim 970 \mathrm{GeV}$. These limits can be pushed to higher values by using more advanced analysis techniques with full simulation of the detectors when the FCC-hh begins to operate.

The signal significances are presented also in tabular form in Table 5 for each $m_{h_{2}}$ value considered in the analysis. (For FCC-hh, also event yields are shown.) As $m_{h_{2}}$ increases, the signal significance gradually drops. This is true for both colliders. Higher luminosities would be needed to explore the multi-TeV mass region as the signal significance drops below $1 \sigma$ already at $\sim 1200 \mathrm{GeV}$ for FCC-hh.

The signal significance as a function of integrated luminosity at $100 \mathrm{TeV}$ FCC-hh for $m_{h_{2}}=800,1000$, and 1200
$\mathrm{GeV}$ is given in Fig. 14. The results for the higher mass values are not shown since the values are too small. This figure ensures that if the mass of $h_{2}$ is about $800 \mathrm{GeV}$, the integrated luminosity required for a $5 \sigma$ discovery is $5.2 \mathrm{ab}^{-1}$. In addition, the results show that an evidence of $h_{2}$ with significance of $3 \sigma$ at the FCC-hh requires $2 \mathrm{ab}^{-1}$ integrated luminosity. This means that FCC-hh offers immediate discovery potential and even at the early stages of its operating period, we can start testing experimentally the BSM sector of symmergence.

These simulation studies show that BSM models that do not destabilize the electroweak scale (such as symmergence) can be probed at relatively high luminosities as their couplings to the SM fields follow the seesawic scheme in (7) and (13). 


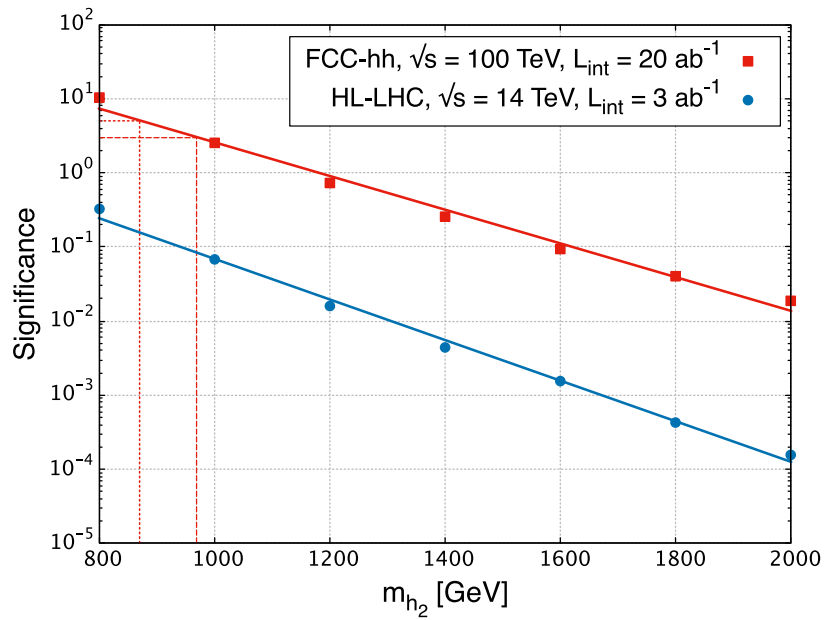

Fig. 13 Significance of the signals calculated as defined in (25) as a function of $m_{h_{2}}$ for FCC-hh with an integrated luminosity $\mathrm{L}_{\text {int }}=$ $20 \mathrm{ab}^{-1}$ (a) and for HL-LHC with an integrated luminosity $\mathrm{L}_{\text {int }}=$ $3 \mathrm{ab}^{-1}$. The red dashed and red dotted lines indicate upper limits on $m_{h_{2}}$ for $3 \sigma$ evidence and $5 \sigma$ discovery, respectively, for FCC-hh at $\mathrm{L}_{\mathrm{int}}=20 \mathrm{ab}^{-1}$

Table 5 Signal significances for HL-LHC and FCC-hh. For FCC-hh, also event yields are shown

\begin{tabular}{lllll}
\hline$m_{h_{2}}(\mathrm{GeV})$ & HL-LHC & FCC-hh & & \\
\cline { 3 - 5 } & $n$ & $n$ & $\mathrm{~S}$ & $\mathrm{~B}$ \\
\hline 800 & 0.326 & 10.36 & 778 & 4857 \\
1000 & 0.067 & 2.533 & 154 & 3541 \\
1200 & 0.016 & 0.733 & 40 & 2935 \\
1400 & 0.004 & 0.254 & 12 & 2212 \\
1600 & 0.001 & 0.093 & 4 & 1993 \\
1800 & $4 \mathrm{e}-4$ & 0.040 & 2 & 1612 \\
2000 & $1 \mathrm{e}-4$ & 0.019 & 0.6 & 1296 \\
\hline
\end{tabular}

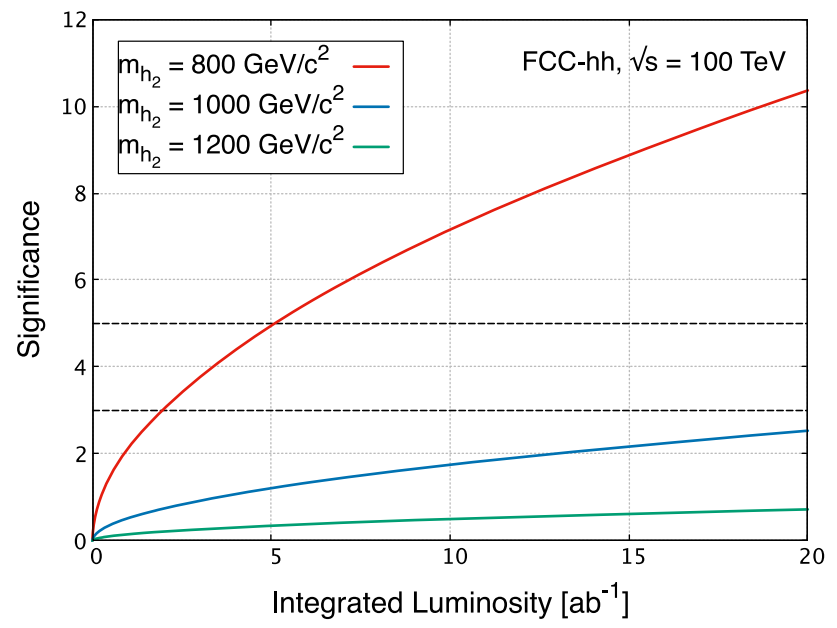

Fig. 14 Signal significance as a function of integrated luminosity at $100 \mathrm{TeV}$ FCC-hh for $m_{h_{2}}=800,1000$ and $1200 \mathrm{GeV}$. The dashed lines correspond $3 \sigma$ and $5 \sigma$ values

\section{Conclusion}

In the present work we have studied impact of the electroweak stability on the collider discovery of the BSM physics, with the example of a single SM-singlet scalar. The discussions in Sect. 2 and the simulation studies in Sect. 5 have revealed that fixing the SM-BSM coupling $\lambda_{H S}$ as in (22) (as a result of the MDDU structure in (13)) has important implications for new particle searches. This coupling fix, admissible in symmergence, tells us that there can exist heavy particles like $h_{2}$ and they can directly couple to the SM Higgs boson but they do not destabilize the electroweak scale thanks to see-sawic structure in (22). Though there is no apparent symmetry structure scalar field theories to support it, the empirical seesawic coupling (22) finds a rationale in the MDDU scheme in (13).

The discovery potentials of $h_{2}$ in proton-proton collisions at the center-of-mass energies of 14 and $100 \mathrm{TeV}$ are studied by analyzing $p p \rightarrow h_{2} \rightarrow Z Z \rightarrow 4 l$ channel, with $3 \mathrm{ab}^{-1}$ total integrated luminosity for the HL-LHC and $20 \mathrm{ab}^{-1}$ for the future circular collider FCC-hh. Seven different mass scenarios for $h_{2}$, between $800-2000 \mathrm{GeV}$ with increments of $200 \mathrm{GeV}$, are considered for the searches. The detector effects are simulated via fast simulation package Delphes. Events with two opposite-sign, same-flavor lepton pair in the final state are selected and the two $\mathrm{Z}$ boson candidates are reconstructed event by event from the lepton pairs. Various observables, transverse momenta of the leptons, mass and transverse momenta of the reconstructed $\mathrm{Z}$ bosons, and invariant mass of the four-lepton system, are used to separate the signal from the background. The signal significances are calculated for each mass value considered in the analysis.

For the HL-LHC with an expected integrated luminosity $3 \mathrm{ab}^{-1}$, no significant excess of signal over the background was observed in the $800 \mathrm{GeV}<m_{h_{2}}<2000 \mathrm{GeV}$ mass range. The highest significance is $0.3 \sigma$ at $800 \mathrm{GeV}$ and gradually drops to $0.0001 \sigma$ at $2000 \mathrm{GeV}$. With higher collision energies and increased luminosities at the FCC-hh (at $100 \mathrm{TeV}$ with $\mathrm{L}_{\text {int }}=20 \mathrm{ab}^{-1}$ ), $h_{2}$ can be discovered with significance of $5 \sigma$ and a mass up to $\sim 870 \mathrm{GeV}$. If the mass of $h_{2}$ is about $800 \mathrm{GeV}$, the integrated luminosity required for a $5 \sigma$ discovery is $5.2 \mathrm{ab}^{-1}$. In addition, the results show that an evidence for $h_{2}$ with significance of $3 \sigma$ at the FCC-hh requires $2 \mathrm{ab}^{-1}$ of integrated luminosity. This means that even at the early stages of the FCC-hh operating period, we can start testing experimentally the BSM sector of symmergence. Considering $20 \mathrm{ab}^{-1}$, the mass of $h_{2}$ can go up to a maximum of $\sim 970 \mathrm{GeV}$ for a $3 \sigma$ evidence. Higher integrated luminosities or searches for $h_{2}$ in different decay channels can push the limits forward, but this is beyond the scope of the analysis presented in this study.

It would be complementary to mention why lower mass values have not been analyzed. The main goal of the work is 
to stabilize the SM against heavy BSM fields and determine under what energy and luminosity ranges that heavy BSM can be discovered. With the example of an SM-singlet scalar, we have found that a seesawic coupling (supported by the MDDU linkup, as revealed in Sect. 2.2) does the job. By the nature of the seesawic coupling (which behaves as $\lambda_{H S} \rightsquigarrow$ $\lambda_{S M}$ when $m_{S}^{2} \rightsquigarrow m_{H}^{2}$ ), however, light scalars, $m_{S}^{2} \rightsquigarrow m_{H}^{2}$, are expected to lead to SM-sized signal strengths. This low$m_{S}$ domain is the one where the known SM completions like supersymmetry work, and the LHC data has already sidelined them for certain parameter regions [59-63]. It is in this sense that our analyses have concentrated on heavy BSM rather than light BSM.

Our analyses and discussions are based on the luminosities and center-of-mass energies of the existing and planned (namely, the FCC) colliders. We have shown that the BSM (represented in this work by a single SM-single scalar $S$ ) can be probed properly in certain channels. It is clear that if these colliders had larger luminosities we would be able to access higher-mass $h_{2}$ bosons at similar levels of significance. Characteristically, electroweak stability necessitates seesawic couplings between the SM and the BSM, and seesawic couplings necessitate high luminosities for discovering the BSM. In domains where a BSM would be excluded with $\mathcal{O}(1)$ couplings (as in supersymmetry, for instance) one can finds room for seesawically-coupled BSM. It is with much higher luminosities that colliders can access multi-TeV BSM, as exemplified by only "TeV mass reach" of the planned FCC-hh collider.

The discussions in the text have concentrated exclusively on the SM-singlet scalar $S$. The reason for this choice, as was mentioned in item (b) in Introduction, is that scalars form the "worst case" when it comes to electroweak stability since not only the Higgs mass but also the scalar mass are quadratically sensitive to the UV cutoff $\Lambda$. Their concurrent stabilization is possible in symmergence, and this has been utilized in the analyses in Sect. 2 and onward. But, a singlet scalar, though not strongly constrained by precision measurements and flavor physics, is difficult to search at colliders. To this end, the hypercharge and lepton portals in (2) could be more promising. In fact, electroweak stability is expected to allow for much heavier $Z^{\prime}$ boson and right-handed neutrinos $N$ when $\left|\lambda_{H S}\right| \sim\left|\lambda_{Z^{\prime} B}\right| \sim\left|\lambda_{L N}\right|$ since $Z^{\prime}$ and $N$ contributions to Higgs mass are quadratic in the couplings, as given in (2). Nevertheless, these rough estimates need be made precise by including all the available bounds. For instance, electroweak stability and known masses of active neutrinos together require the right-handed neutrinos to weigh below a $1000 \mathrm{TeV}$ [7]. It thus follows that the present work need be extended to the $Z^{\prime}$ and $N$ sectors by incorporating available bounds (neutral currents, neutrino masses, flavor physics). Their collider analyses, left to future work, can reveal important search strategies and distinctive signatures at future colliders like the LHC and FCC.

Acknowledgements This work is supported in part by the TÜBİTAK grant 118 F387 and by the ITU BAP grant TAB-2020-42312. We thank Beyhan Puliçe for her contributions at the initial stage of this work. We are grateful to conscientious referee for her/his constructive comments, criticisms and suggestions.

Data Availability Statement This manuscript has no associated data or the data will not be deposited. [Authors' comment: This is a phenomenological study, and no experimental data were generated.]

Open Access This article is licensed under a Creative Commons Attribution 4.0 International License, which permits use, sharing, adaptation, distribution and reproduction in any medium or format, as long as you give appropriate credit to the original author(s) and the source, provide a link to the Creative Commons licence, and indicate if changes were made. The images or other third party material in this article are included in the article's Creative Commons licence, unless indicated otherwise in a credit line to the material. If material is not included in the article's Creative Commons licence and your intended use is not permitted by statutory regulation or exceeds the permitted use, you will need to obtain permission directly from the copyright holder. To view a copy of this licence, visit http://creativecomm ons.org/licenses/by/4.0/.

Funded by $\mathrm{SCOAP}^{3}$.

\section{Appendix}

The vertex factors in (20) have the following expressions:

$$
\begin{aligned}
& \lambda_{h_{1} h_{1} h_{1} h_{1}}=\frac{\lambda_{H}}{4} \cos ^{4} \theta+\frac{\lambda_{S}}{4} \sin ^{4} \theta+\frac{\lambda_{H S}}{16} \sin ^{2} 2 \theta \\
& \lambda_{h_{1} h_{1} \phi \phi}=\lambda_{h_{1} h_{1} \phi_{0} \phi_{0}}=\lambda_{h_{1} h_{1} \phi_{1} \phi_{1}}=\lambda_{h_{1} h_{1} \phi_{2} \phi_{2}} \\
& =\frac{\lambda_{H}}{2} \cos ^{2} \theta+\frac{\lambda_{H S}}{4} \sin ^{2} \theta \\
& \lambda_{h_{1} h_{1} h_{2} h_{2}}=\frac{3}{8}\left(\lambda_{H}+\lambda_{S}\right) \sin ^{2} 2 \theta \\
& +\frac{\lambda_{H S}}{4}\left(\cos ^{4} \theta+\sin ^{4} \theta-\sin ^{2} 2 \theta\right) \\
& \lambda_{h_{1} h_{1} h_{1}}=\lambda_{H} v_{H} \cos ^{3} \theta-\lambda_{S} v_{S} \sin ^{3} \theta \\
& -\frac{\lambda_{H S}}{4}\left(\cos \theta v_{S}-\sin \theta v_{H}\right) \sin 2 \theta \\
& \lambda_{h_{1} \phi \phi}=\lambda_{h_{1} \phi_{0} \phi_{0}}=\lambda_{h_{1} \phi_{1} \phi_{1}}=\lambda_{h_{1} \phi_{2} \phi_{2}} \\
& =\lambda_{H} v_{H} \cos \theta-\frac{\lambda_{H S}}{2} v_{S} \sin \theta \\
& \lambda_{h_{1} h_{2} h_{2}}=\frac{3}{2}\left(\lambda_{H} v_{H} \sin \theta-\lambda_{S} v_{S} \cos \theta\right) \sin 2 \theta \\
& +\frac{\lambda H S}{2}\left(\left(\cos \theta \sin 2 \theta-\sin ^{3} \theta\right) v_{S}\right. \\
& \left.+\left(\cos ^{3} \theta-\sin \theta \sin 2 \theta\right) v_{H}\right) \\
& \lambda_{h_{1} h_{1} h_{2}}=\frac{3}{2}\left(\lambda_{H} v_{H} \cos \theta+\lambda_{S} v_{S} \sin \theta\right) \sin 2 \theta \\
& +\frac{\lambda_{H S}}{2}\left(\left(\cos ^{3} \theta-\sin \theta \sin 2 \theta\right) v_{S}\right. \\
& \left.+\left(\sin ^{3} \theta-\cos \theta \sin 2 \theta\right) v_{H}\right) \text {. }
\end{aligned}
$$




\section{References}

1. G. Aad et al. (ATLAS and CMS Collaborations), JHEP 1608, 045 (2016). arXiv:1606.02266 [hep-ex]

2. M. Tanabashi et al. (Particle Data Group), Phys. Rev. D 98, 030001 (2018)

3. B. Vachon (ATLAS and CMS Collaborations), Int. J. Mod. Phys. A 31, 1630034 (2016)

4. C. Csaki, C. Grojean, J. Terning, Rev. Mod. Phys. 88, 045001 (2016). arXiv:1512.00468 [hep-ph]

5. C. Csaki, P. Tanedo, Beyond the standard model. arXiv:1602.04228 [hep-ph]

6. D. Demir, arXiv:1703.05733 [hep-ph]

7. D. Demir, Adv. High Energy Phys. 2019, 4652048 (2019). arXiv:1901.07244 [hep-ph]

8. R. Barbieri, A. Strumia, The 'LEP Paradox'. arXiv:hep-ph/0007265

9. A. Birkedal, Z. Chacko, M.K. Gaillard, JHEP 0410, 036 (2004). arXiv:hep-ph/0404197

10. R. Foot, A. Kobakhidze, K.L. McDonald, R.R. Volkas, Phys. Rev. D 89, 115018 (2014). arXiv:1310.0223 [hep-ph]

11. L. Susskind, Phys. Rev. D 20, 2619 (1979)

12. M.J.G. Veltman, Acta Phys. Pol. B 12, 437 (1981)

13. G.F. Giudice, PoS EPS 163 (2013)

14. D.A. Demir, Adv. High Energy Phys. 2016, 6727805 (2016). arXiv:1605.00377 [hep-ph]

15. M.E. Peskin, D.V. Schroeder, An Introduction to Quantum Field Theory (Addison-Wesley, Reading, 1995)

16. M. D’Attanasio, T.R. Morris, Phys. Lett. B 378, 213 (1996). arXiv:hep-th/9602156

17. P.H. Chankowski, A. Lewandowski, K.A. Meissner, Acta Phys. Pol. B 48, 5 (2017). arXiv:1608.01214 [hep-th]

18. J. Polchinski, Nucl. Phys. B 231, 269-295 (1984)

19. G. 't Hooft, M. Veltman, Nucl. Phys. B 44, 189-213 (1972)

20. W.A. Bardeen, On naturalness in the standard model, FERMILABCONF-95-391-T (1995)

21. K.A. Meissner, H. Nicolai, Phys. Lett. B 648, 312-317 (2007). arXiv:hep-th/0612165

22. G. Marques Tavares, M. Schmaltz, W. Skiba, Phys. Rev. D 89, 015009 (2014). arXiv:1308.0025 [hep-ph]

23. Z. Chacko, H.S. Goh, R. Harnik, Phys. Rev. Lett. 96, 231802 (2006). arXiv:hep-ph/0506256

24. R. Contino, D. Greco, R. Mahbubani, R. Rattazzi, R. Torre, Phys. Rev. D 96(9), 095036 (2017). arXiv:1702.00797 [hep-ph]

25. P.W. Graham, D.E. Kaplan, S. Rajendran, Phys. Rev. Lett. 115, 221801 (2015). arXiv:1504.07551 [hep-ph]

26. B. Batell, G.F. Giudice, M. McCullough, JHEP 1512, 162 (2015). arXiv:1509.00834 [hep-ph]

27. D.A. Demir, Gravi-natural Higgs and conformal new physics. arXiv:1207.4584 [hep-ph]

28. H. Matsui, Y. Matsumoto, Gravitational relaxation of electroweak hierarchy problem. arXiv:1608.08838 [hep-ph]

29. G. Dvali, Fortschr. Phys. 58, 528 (2010). arXiv:0706.2050 [hep-th]

30. N. Arkani-Hamed, T. Cohen, R.T. D'Agnolo, A. Hook, H.D. Kim, D. Pinner, Phys. Rev. Lett. 117, 251801 (2016). arXiv:1607.06821 [hep-ph]

31. H. Aoki, S. Iso, Phys. Rev. D 86, 013001 (2012). arXiv:1201.0857 [hep-ph]

32. S. Davidson, H.E. Haber, Phys. Rev. D 72, 035004 (2005). arXiv:hep-ph/0504050

33. H.E. Haber, D. O’Neil, Phys. Rev. D 83, 055017 (2011). arXiv:1011.6188 [hep-ph]
34. I.F. Ginzburg, M. Krawczyk, Phys. Rev. D 72, 115013 (2005). arXiv:hep-ph/0408011

35. P.M. Ferreira, J.P. Silva, Phys. Rev. D 83, 065026 (2011). arXiv:1012.2874 [hep-ph]

36. G. Giudice, A. Masiero, Phys. Lett. B 206, 480-484 (1988)

37. D. Demir, C.S. Un, Scalar dark matter and electroweak stability. arXiv:2005.03589 [hep-ph]

38. J.D. Bjorken, Int. J. Mod. Phys. A 7, 4189 (1992)

39. D.A. Demir, Phys. Lett. B 450, 215 (1999). arXiv:hep-ph/9810453

40. G.C. Branco, P.M. Ferreira, L. Lavoura, M.N. Rebelo, M. Sher, J.P Silva, Phys. Rep. 516, 1 (2012). arXiv:1106.0034 [hep-ph]

41. C.Y. Chen, S. Dawson, M. Sher, Phys. Rev. D 88, 015018 (2013). arXiv:1305.1624 [hep-ph] [Erratum: Phys. Rev. D 88, 039901 (2013)]

42. D. Alves et al. (LHC New Physics Working Group), J. Phys. G 39, 105005 (2012). arXiv:1105.2838 [hep-ph]

43. G. Aad et al. (ATLAS), Phys. Lett. B 718, 391-410 (2012). arXiv:1206.6074 [hep-ex]

44. G. Aad et al. (ATLAS), Phys. Lett. B 798, 134949 (2019). arXiv:1903.10052 [hep-ex]

45. G. Aad et al. (ATLAS), Phys. Rev. Lett. 112(23), 231806 (2014). arXiv: 1403.5657 [hep-ex]

46. G. Aad et al. (ATLAS), Phys. Lett. B 712, 331-350 (2012). arXiv:1203.0718 [hep-ex]

47. M. Aleksa et al., arXiv:1912.09962 [physics.ins-det]

48. B. Schmidt, J. Phys. Conf. Ser. 706(2), 022002 (2016)

49. ATLAS and CMS Collaborations (ATLAS and CMS Collaborations), CERN Yellow Rep. Monogr. 7 (2019). arXiv:1902.10229 [hep-ex] [Addendum]

50. A. Semenov, Comput. Phys. Commun. 201, 167 (2016). arXiv:1412.5016 [physics.comp-ph]

51. A. Belyaev, N.D. Christensen, A. Pukhov, Comput. Phys. Commun. 184, 1729 (2013). arXiv:1207.6082 [hep-ph]

52. A. Buckley, J. Ferrando, S. Lloyd, K. Nordstrom, B. Page, M. Rafenacht, M. Schonherr, G. Watt, Eur. Phys. J. C 75, 132 (2015). arXiv: 1412.7420 [hep-ph]

53. J. Pumplin, D.R. Stump, J. Huston, H.L. Lai, P.M. Nadolsky, W.K. Tung, JHEP 0207, 012 (2002). arXiv:hep-ph/0201195

54. T. Sjöstrand, S. Ask, J.R. Christiansen, R. Corke, N. Desai, P. Ilten, S. Mrenna, S. Prestel, C.O. Rasmussen, P.Z. Skands, Comput. Phys. Commun. 191, 159-177 (2015). arXiv:1410.3012 [hep-ph]

55. J. de Favereau et al. (DELPHES 3), JHEP 02, 057 (2014). https:// doi.org/10.1007/JHEP02(2014)057. arXiv:1307.6346 [hep-ex]

56. I. Antcheva et al., Comput. Phys. Commun. 180, 2499-2512 (2009). arXiv:1508.07749 [physics.data-an]

57. A.M. Sirunyan et al. (CMS), Eur. Phys. J. C 78, 165 (2018). arXiv:1709.08601 [hep-ex]

58. S. Banerjee, M. Mitra, M. Spannowsky, Phys. Rev. D 92(5), 055013 (2015). arXiv:1506.06415 [hep-ph]

59. G. Aad et al. (ATLAS), JHEP 11, 056 (2014). https://doi.org/10. 1007/JHEP11(2014)056. arXiv:1409.6064 [hep-ex]

60. V. Khachatryan et al. (CMS), JHEP 11, 071 (2015). https://doi.org/ 10.1007/JHEP11(2015)071. arXiv:1506.08329 [hep-ex]

61. A.M. Sirunyan et al. (CMS), Eur. Phys. J. C 78(4), 291 (2018). https://doi.org/10.1140/epjc/s10052-018-5740-1. arXiv:1711.00431 [hep-ex]

62. A.M. Sirunyan et al. (CMS), Phys. Lett. B 798, 134992 (2019). https://doi.org/10.1016/j.physletb.2019.134992. arXiv:1907.03152 [hep-ex]

63. A.M. Sirunyan et al. (CMS), JHEP 04, 171 (2020). https://doi.org/ 10.1007/JHEP04(2020)171. arXiv:1908.01115 [hep-ex] 